\title{
ENTROPY IN THE CUSP AND PHASE TRANSITIONS FOR GEODESIC FLOWS
}

\author{
GODOFREDO IOMMI, FELIPE RIQUELME, AND ANIBAL VELOZO
}

\begin{abstract}
In this paper we study the geodesic flow for a particular class of Riemannian non-compact manifolds with variable pinched negative sectional curvature. For a sequence of invariant measures we are able to prove results relating the loss of mass and bounds on the measure entropies. We compute the entropy contribution of the cusps. We develop and study the corresponding thermodynamic formalism. We obtain certain regularity results for the pressure of a class of potentials. We prove that the pressure is real analytic until it undergoes a phase transition, after which it becomes constant. Our techniques are based on the one side on symbolic methods and Markov partitions and on the other on geometric techniques and approximation properties at level of groups.
\end{abstract}

\section{INTRODUCTION}

This paper is devoted to study thermodynamic formalism for a class of geodesic flows defined over non-compact manifolds of variable pinched negative curvature. These flows can be coded with suspension flows defined over Markov shifts, albeit on a countable alphabet. This paper addresses two different but related problems where the non-compactness of the ambient manifold plays a fundamental role. Inspired in some recent results proved in the context of homogeneous dynamics ([ELMV, EKP]), we establish properties that relate the escape of mass of a sequence of invariant probability measures for the geodesic flow with its measure theoretic entropies (see section 5). The second goal of the paper is to construct a class of potentials for which the pressure exhibits a phase transition (see section 6). To obtain these results we combine both geometric and symbolic methods.

The class of manifolds that we will be working on along the paper were introduced in [DP]. These manifolds are obtained as the quotient of a Hadamard manifold with an extended Schottky group (see sub-section 4.2 for precise definitions). This class of groups have parabolic elements of rank 1 , therefore the manifolds are noncompact. It was shown in [DP] that the geodesic flow over the unit tangent bundle of those manifolds can be coded as suspension flows over countable Markov shifts. The existence of a Markov coding for the geodesic flow is essential for ours results.

The idea of coding a flow in order to describe its dynamical and ergodic properties has long history and a great deal of interesting and important results have been obtained with these methods. Probably, some of the most relevant results using this

Date: January 28, 2016.

2010 Mathematics Subject Classification. 15A15; 26E60, 37A30, 60B20, 60F15.

G.I. was partially supported by the Center of Dynamical Systems and Related Fields código ACT1103 and by Proyecto Fondecyt 1150058.

F.R. was supported by Programa de Cooperación Científica Internacional CONICYT-CNRS código PCCI 14009. 
technique are related to counting closed geodesics and also estimating the rate at which they grow [PP]. A landmark result is the construction of Markov partitions for Axiom A flows defined over compact manifolds done by Bowen [Bo1] and Ratner [Ra]. They actually showed that Axiom A flows can be coded with suspension flows defined over sub-shifts of finite type on finite alphabets with a regular (Hölder) roof functions. The study in the non-compact setting is far less developed. However, some interesting results have been obtained. Recently, Hamenstädt [H] and also Bufetov and Gurevich [BG] have coded Teichmüller flows with suspension flows over countable alphabets and using this representation have proved, for example, the uniqueness of the measure of maximal entropy. Another important example for which codings on countable alphabets have been constructed is a type of Sinai billiards [BS1, BS2].

As mentioned before, one goal of the paper is to investigate the loss of mass of sequences of invariant measures for the geodesic flow. Recently, the loss of mass has been studied for the modular surface in [ELMV]. Despite being a particular case, the method displayed in [ELMV] is quite flexible and has the advantage it can be understood purely geometrically. A more general situation is studied in [EKP], the results apply to any geodesic flow on a finite volume hyperbolic surface (with respect to the constant sectional curvature metric). We begin introducing the notion of entropy at infinity of a dynamical system defined over a non-compact topological space.

Definition 1.1. Let $Y$ be a non-compact topological space and $S=\left(S_{t}\right)_{t \in \mathbb{R}}: Y \rightarrow Y$ a continuous flow. We define the "entropy at infinity" of the dynamical system as the number

$$
h_{\infty}\left(Y,\left\{S_{t}\right\}\right)=\sup _{\left\{\nu_{n}\right\} \rightarrow 0} \limsup _{n \rightarrow \infty} h_{\nu_{n}}(S),
$$

where the supremum is taken over all the sequences of invariant probability measures for the flow converging in the vague topology to the zero measure. If no such sequence exists we set $h_{\infty}\left(Y,\left\{S_{t}\right\}\right)=0$. Here $h_{\nu}(S)$ denotes the measure-theoretic entropy of a probability $S$-invariant measure $\nu$.

Recall that the total mass of probability measures is not necessarily preserved under vague convergence (as opposite to weak convergence). Note that Definition 1.1 can be extended to more general group actions whenever an entropy theory has been developed for the group in consideration. Amenable groups are a classical example of such.

In this paper we are able to compute $h_{\infty}\left(T^{1} X / \Gamma,\left(g_{t}\right)\right)$, where $X$ is a Hadamard manifold with pinched negative sectional curvature, $\Gamma$ is an extended Schottky group generated by $N_{1}$ hyperbolic isometries and $N_{2}$ parabolic ones, and $\left(g_{t}\right)$ is the geodesic flow on the unit tangent bundle $T^{1} X / \Gamma$ (see sub-section 4.2 for precise definitions). Define

$$
\delta_{p, \max }:=\max \left\{\delta_{p_{i}}, 1 \leqslant i \leqslant N_{2}\right\},
$$

where $\delta_{p_{i}}$ is the critical exponent of the Poincaré series of the group $<p_{i}>$ and $p_{i}$ is a parabolic element of $\Gamma$. We prove that

$$
h_{\infty}\left(T^{1} X / \Gamma,\left(g_{t}\right)\right)=\delta_{p, \max } .
$$

It worth mentioning $\delta_{p, \max }$ is strictly less than the topological entropy of the geodesic flow. In our context, the non-compact pieces of our space are modeled by cusps. That is why we refer to this quantity as entropy in the cusps. More concretely 
we prove that if a sequence of measures are dissipating through the cusps, then the entropy contribution of the sequence is at most $\delta_{p, \max }$. In [EKP] it is proven that $h_{\infty}(\Gamma \backslash G, A)=h_{\text {top }} / 2$ where $G$ is a connected semisimple Lie group of real rank 1 with finite center, $\Gamma$ a lattice in $G$ and $A$ a one-parameter subgroup of diagonalizable elements over $\mathbb{R}$ acting by right multiplication. In particular $h_{\infty}\left(T^{1} S,\left(g_{t}\right)\right)=1 / 2$, where $S$ is a hyperbolic surface with finite volume. We also obtain results in the case where the sequence of measures keep some mass at the limit. Our bounds are less concrete than the analog result in the homogeneous dynamical case though. The following is one of our main results and gives the calculation of the entropy in the cusps mentioned before. The manifold under consideration is assumed to satisfy some mild technical conditions, denoted by $(\star)$. This condition is explained in detail in subsection 4.2.

Theorem 1.2. Let $X$ be a Hadamard manifold with pinched negative sectional curvature and let $\Gamma$ be an extended Schottky group of isometries of $X$ with property $(\star)$. Assume that the derivatives of the sectional curvature are uniformly bounded. Then, for every $c>\delta_{p, \max }$ there exists a constant $m=m(c)>0$, with the following property: If $\left(\nu_{n}\right)$ is a sequence of $\left(g_{t}\right)$-invariant probability measures on $T^{1} X / \Gamma$ satisfying $h_{\nu_{n}}(g) \geqslant c$, then for every vague limit $\nu_{n} \rightarrow \nu$, we have

$$
\nu\left(T^{1} X / \Gamma\right) \geqslant m .
$$

In particular if $\nu_{n} \rightarrow 0$ then $\lim \sup h_{\nu_{n}}(g) \leqslant \delta_{p, \max }$. Moreover, the value $\delta_{p, \max }$ is optimal in the following sense: there exists a sequence $\left(\nu_{n}\right)$ of $\left(g_{t}\right)$-invariant probability measures on $T^{1} X / \Gamma$ such that $h_{\nu_{n}}(g) \rightarrow \delta_{p, \max }$ and $\nu_{n} \rightarrow 0$.

We believe similar results can be obtained for the geodesic flow in the geometrically finite case (maybe under some strong conditions on the curvature).

Our second goal is to study regularity properties of the pressure function. In order to do so, we make strong use of the symbolic coding that the geodesic flow has in the manifolds we are considering. The idea of using symbolic dynamics to study thermodynamic formalism of flows of geometric nature can be traced back to the work of Bowen and Ruelle [BR]. They studied in great detail ergodic theory and thermodynamic formalism for Axiom A flows defined on compact manifolds. The techniques they used were symbolic in nature and were based on the symbolic codings obtained by Bowen [Bol] and Ratner [Ra]. In this work we follow this strategy. We stress, however, that our symbolic models are non-compact. There are several difficulties related to the lack of compactness that have to be addressed, but also new phenomena are observed.

To begin with, in sub-section 6 we propose a definition of topological pressure, $P(\cdot)$, that satisfies not only the variational principle, but also an approximation by compact invariant sets property. These provide symbolic proofs to results obtained by different (non-symbolic) methods in far more general settings by Paulin, Pollicott and Schapira [PPS]. The strength of our approach is perhaps better appreciated in our regularity results for the pressure (sub-section 6.2). Note that the techniques in $[\mathrm{PPS}]$ do not provide these type of results. We say that the pressure function $t \mapsto P(t f)$ has a phase transition at $t=t_{0}$ if it is not analytic at that point. It readily follows from work by Bowen and Ruelle $[\mathrm{BR}]$ that the pressure for Axiom A flows and regular potentials is real analytic and hence has no phase transitions. Regularity properties of the pressure of geodesic flows defined on non-compact 
manifolds, as far as we know, have not been studied, with the exception of the geodesic flow defined on the modular surface (see [IJ, Section 6]).

There is a general strategy used to study regularity properties of the pressure of maps and flows with strong hyperbolic or expanding properties in most of the phase space but not in all of it. Indeed, if there exists a subset of the phase space $B \subset X$ for which the restricted dynamics is not expansive and its entropy equal to $A$, then it is possible to construct potentials $f: X \rightarrow \mathbb{R}$ for which the pressure function has the form

$$
P(t f):= \begin{cases}\text { real analytic, strictly decreasing and convex } & \text { if } t<t^{\prime} \\ A & \text { if } t>t^{\prime}\end{cases}
$$

Well known examples of this phenomena include the Manneville-Pomeau map (see for example [Sa2]) in which the set $B$ consist of a parabolic fixed point and therefore $A=0$. The potential considered is the geometrical one: $-\log \left|T^{\prime}\right|$. Similar results for multimodal maps have been obtained, for example, in [DT, IT1, PR]. In this case the set $B$ corresponds to the post-critical set and $A=0$. Examples of maps in which $A>0$ have been studied in [DGR, IT2]. For suspension flows over countable Markov shifts, similar examples were obtained in [IJ]. However, in that case the number $A$, which we denote by $s_{\infty}$, remained unexplained for. In this paper we show that the entropy at infinity of a suspension flow over a countable Markov shift corresponds to $s_{\infty}$ (see Corollary 3.8).

In the case of geodesic flows, roughly speaking we are considering the set $B$ as the union of the cusps of the manifold. More interestingly, as we mentioned before we are able to compute the entropy contributions of the cusps in the geodesic flow. In sub-section 6.2 we construct a class of potentials, that we denote by $\mathcal{F}$, for which the pressure exhibits similar behaviour as in equation (1). In those examples $A=\delta_{p, \max }$. Note that it is possible for $t^{\prime}$ to be infinity and in that case the pressure is real analytic. The following is the precise statement,

Theorem 1.3. Let $X$ be a Hadamard manifold with pinched negative sectional curvature and let $\Gamma$ be an extended Schottky group of isometries of $X$ with property $(\star)$. Assume that the derivatives of the sectional curvature are uniformly bounded. If $f \in \mathcal{F}$, then

(1) For every $t \in \mathbb{R}$ we have that $P_{g}(t f) \geqslant \delta_{p, \max }$.

(2) We have that $\lim _{t \rightarrow-\infty} P_{g}(t f)=\delta_{p, \max }$.

(3) Let $t^{\prime}:=\sup \left\{t \in \mathbb{R}: P_{g}(t f)=\delta_{p, \max }\right\}$, then

$$
P_{g}(t f)= \begin{cases}\delta_{p, \max } & \text { if } t<t^{\prime} \\ \text { real analytic, strictly convex, strictly increasing } & \text { if } t>t^{\prime} .\end{cases}
$$

(4) If $t>t^{\prime}$, the potential tf has a unique equilibrium measure. If $t<t^{\prime}$ it has no equilibrium measure.

In order to prove this result we need to relate symbolic quantities with geometrical ones. This is achieved in Theorem 4.12 in which a symbolic parameter of the suspension flow, the number $s_{\infty}$, is proven to be equal to the geometric parameter of the group $\delta_{p, \max }$. We stress that when coding a flow a great deal of geometric information is lost. With this result we are able to recover part of it. 
Acknowledgements. We thank B. Schapira for a careful reading of the paper and for several interesting and useful comments. We also thank F. Dal'bo and F. Ledrappier for several enlighten and interesting discussions around the subject treated in this article. This work started when the second and third authors were visiting Pontificia Universidad Católica de Chile, they are very grateful for the great conditions and hospitality received in PUC Mathematics Department.

\section{PReliminaries on thermodynamic FORMALISM AND SUSPENSION FlOWS}

This section is devoted to provide the necessary background on thermodynamic formalism and on suspension flows required on the rest of the article.

2.1. Thermodynamic formalism for countable Markov shifts. Let $M$ be an incidence matrix defined on the alphabet of natural numbers. The associated one sided countable Markov shift $\left(\Sigma^{+}, \sigma\right)$ is the set

$$
\Sigma^{+}:=\left\{\left(x_{n}\right)_{n \in \mathbb{N}}: M\left(x_{n}, x_{n+1}\right)=1 \text { for every } n \in \mathbb{N}\right\},
$$

together with the shift map $\sigma: \Sigma^{+} \rightarrow \Sigma^{+}$defined by $\sigma\left(x_{1}, x_{2}, \ldots\right)=\left(x_{2}, x_{3}, \ldots\right)$. A standing assumption we will make throughout the article is that $\left(\Sigma^{+}, \sigma\right)$ is topologically mixing. We equip $\Sigma^{+}$with the topology generated by the cylinder sets

$$
C_{a_{1} \cdots a_{n}}=\left\{x \in \Sigma^{+}: x_{i}=a_{i} \text { for } i=1, \ldots, n\right\} .
$$

We stress that, in general, $\Sigma^{+}$is a non-compact space. Given a function $\varphi: \Sigma^{+} \rightarrow \mathbb{R}$ we define the $n$-th variations of $\varphi$ by

$$
V_{n}(\varphi):=\sup \left\{|\varphi(x)-\varphi(y)|: x, y \in \Sigma^{+}, x_{i}=y_{i} \text { for } i=1, \ldots, n\right\},
$$

where $x=\left(x_{1} x_{2} \cdots\right)$ and $y=\left(y_{1} y_{2} \cdots\right)$. We say that $\varphi$ has summable variation if $\sum_{n=1}^{\infty} V_{n}(\varphi)<\infty$. We say that $\varphi$ is locally Hölder if there exists $\theta \in(0,1)$ such that for all $n \geqslant 1$, we have $V_{n}(\varphi) \leqslant O\left(\theta^{n}\right)$.

This section is devoted to recall some of the notions and results of thermodynamic formalism in this setting. The following definition was introduced by Sarig [Sa1] based on work by Gurevich [Gu].

Definition 2.1. Let $\varphi: \Sigma^{+} \rightarrow \mathbb{R}$ be a function of summable variation. The Gurevich pressure of $\varphi$ is defined by

$$
P(\varphi)=\lim _{n \rightarrow \infty} \frac{1}{n} \log \sum_{x: \sigma^{n} x=x} \exp \left(\sum_{i=0}^{n-1} \varphi\left(\sigma^{i} x\right)\right) \chi_{C_{i_{1}}}(x),
$$

where $\chi_{C_{i_{1}}}(x)$ is the characteristic function of the cylinder $C_{i_{1}} \subset \Sigma^{+}$.

It is possible to show (see [Sa1, Theorem 1]) that the limit always exists and that it does not depend on $i_{1}$. The following two properties of the pressure will be relevant for our purposes (see [Sa1, Theorems 2 and 3] and [IJT, Theorem 2.10]). If $\varphi: \Sigma^{+} \rightarrow \mathbb{R}$ is a function of summable variations, then

(1) (Approximation property)

$$
P(\varphi)=\sup \left\{P_{K}(\varphi): K \in \mathcal{K}\right\},
$$

where $P_{K}(\varphi)$ is the classical topological pressure on $K$ (see [Wa, Chapter 9]) and

$$
\mathcal{K}:=\left\{K \subset \Sigma^{+}: K \neq \varnothing \text { compact and } \sigma \text {-invariant }\right\} .
$$


(2) (Variational Principle) Denote by $\mathcal{M}_{\sigma}$ is the space of $\sigma$-invariant probability measures and by $h_{\mu}(\sigma)$ the entropy of the measure $\mu$ (see [Wa, Chapter 4]). If $\varphi: \Sigma^{+} \rightarrow \mathbb{R}$ is a function of summable variation then,

$$
P_{\sigma}(\varphi)=\sup \left\{h_{\mu}(\sigma)+\int \varphi \mathrm{d} \mu: \mu \in \mathcal{M}_{\sigma} \text { and }-\int \varphi \mathrm{d} \mu<\infty\right\} .
$$

A measure $\mu \in \mathcal{M}_{\sigma}$ attaining the supremum, that is, $P_{\sigma}(\varphi)=h_{\mu}(\sigma)+\int \varphi \mathrm{d} \mu$ is called equilibrium measure for $\varphi$. A potential of summable variations has at most one equilibrium measure (see [BuS, Theorem 1.1]).

It turns out that under a combinatorial assumption on the incidence matrix $M$, which roughly means to be similar to a full-shift, the thermodynamic formalism is well behaved.

Definition 2.2. We say that a countable Markov shift $\left(\Sigma^{+}, \sigma\right)$, defined by the transition matrix $M(i, j)$ with $(i, j) \in \mathbb{N} \times \mathbb{N}$, satisfies the BIP (Big Images and Preimages) condition if and only if there exists $\left\{b_{1}, \ldots, b_{n}\right\} \subset \mathbb{N}$ such that for every $a \in \mathbb{N}$ there exists $i, j \in \mathbb{N}$ with $M\left(b_{i}, a\right) M\left(a, b_{j}\right)=1$.

The following theorem summarises results proven by Sarig in [Sa1, Sa2] and by Mauldin and Urbański, [MU], where they show that thermodynamic formalism in this setting is similar to that observed for sub-shifts of finite type on finite alphabets.

Theorem 2.3. Let $\left(\Sigma^{+}, \sigma\right)$ be a countable Markov shift satisfying the BIP condition and $\varphi: \Sigma^{+} \rightarrow \mathbb{R}$ a non-positive locally Hölder potential. Then, there exists $s_{\infty}>0$ such that pressure function $t \rightarrow P_{\sigma}(t \varphi)$ has the following properties

$$
P_{\sigma}(t \varphi)= \begin{cases}\infty & \text { if } t<s_{\infty} \\ \text { real analytic } & \text { if } t>s_{\infty} .\end{cases}
$$

Moreover, if $t>s_{\infty}$, there exists a unique equilibrium measure for $t \varphi$.

2.2. Suspension flows. Let $\left(\Sigma^{+}, \sigma\right)$ be a topologically mixing countable Markov shift and $\tau: \Sigma^{+} \rightarrow \mathbb{R}^{+}$a function of summable variations bounded away from zero. Consider the space

$$
Y=\left\{(x, t) \in \Sigma^{+} \times \mathbb{R}: 0 \leqslant t \leqslant \tau(x)\right\},
$$

with the points $(x, \tau(x))$ and $(\sigma(x), 0)$ identified for each $x \in \Sigma^{+}$. The suspension semi-flow over $\sigma$ with roof function $\tau$ is the semi-flow $\Phi=\left(\varphi_{t}\right)_{t \geqslant 0}$ on $Y$ defined by

$$
\varphi_{t}(x, s)=(x, s+t) \text { whenever } s+t \in[0, \tau(x)] .
$$

In particular,

$$
\varphi_{\tau(x)}(x, 0)=(\sigma(x), 0) .
$$

2.3. Invariant measures. Let $(Y, \Phi)$ be a suspension semi-flow defined over a countable Markov shift $\left(\Sigma^{+}, \sigma\right)$ with roof function $\tau: \Sigma^{+} \rightarrow \mathbb{R}^{+}$bounded away from zero. Denote by $\mathcal{M}_{\Phi}$ the space of invariant probability measures for the flow. It follows form a classical result by Ambrose and Kakutani [AK] that every measure $\nu \in \mathcal{M}_{\Phi}$ can be written as

$$
\nu=\frac{\left.(\mu \times m)\right|_{Y}}{(\mu \times m)(Y)},
$$

where $\mu \in \mathcal{M}_{\sigma}$ and $m$ denotes the one dimensional Lebesgue measure. When $\left(\Sigma^{+}, \sigma\right)$ is a sub-shift of finite type defined on a finite alphabet the relation in 
equation (3) is actually a bijection between $\mathcal{M}_{\sigma}$ and $\mathcal{M}_{\Phi}$. If $\left(\Sigma^{+}, \sigma\right)$ is a countable Markov shift with roof function bounded away from zero the map defined by

$$
\nu \mapsto \frac{\left.(\mu \times m)\right|_{Y}}{(\mu \times m)(Y)},
$$

is surjective. However, it can happen that $(\mu \times m)(Y)=\infty$. In this case the image can be understood as an infinite invariant measure.

The case which is more subtle is when the roof function is only assumed to be positive. We will not be interested in that case here, but we refer to [IJT] for a discussion on the pathologies that might occur.

2.4. Of flows and semi-flows. In 1972 Sinai [Si, Section 3] observed that in order to study thermodynamic formalism for suspension flows it suffices to study thermodynamic formalism for semi-flows. Denote by $(\Sigma, \sigma)$ a two-sided countable Markov shift. Recall that two continuous functions $\varphi, \gamma \in C(\Sigma)$ are said to be cohomologous if there exists a bounded continuous function $\psi \in C(\Sigma)$ such that $\varphi=\gamma+\psi \circ \sigma-\psi$. The relevant remark is that thermodynamic formalism for two cohomologous functions is exactly the same. Thus, if every continuous function $\varphi \in C(\Sigma)$ is cohomologous to a continuous function $\gamma \in C(\Sigma)$ which only depends in future coordinates then thermodynamic formalism for the flow can be studied in the corresponding semi-flow. The next result formalises this discussion.

Proposition 2.4. If $\varphi \in C(\Sigma)$ has summable variation, then there exists $\gamma \in C(\Sigma)$ of summable variation cohomologous to $\varphi$ such that $\gamma(x)=\gamma(y)$ whenever $x_{i}=y_{i}$ for all $i \geqslant 0$ (that is, $\gamma$ depends only on the future coordinates).

Proposition 2.4 has been proved with different regularity assumptions in the compact setting and in the non-compact case in [Da, Theorem 7.1].

2.5. Abramov and Kac. The entropy of a flow with respect to an invariant measure can be defined as the entropy of the corresponding time one map. The following result was proved by Abramov [Ab].

Proposition 2.5 (Abramov). Let $\nu \in \mathcal{M}_{\Phi}$ be such that $\nu=\left.(\mu \times m)\right|_{Y} /(\mu \times m)(Y)$, where $\mu \in \mathcal{M}_{\sigma}$ then the entropy of $\nu$ with respect to the flow, that we denote $h_{\nu}(\Phi)$, satisfies

$$
h_{\nu}(\Phi)=\frac{h_{\mu}(\sigma)}{\int \tau d \mu}
$$

In Proposition 2.5 a relation between the entropy of a measure for the flow and a corresponding measure for the base dynamics was established. We now prove a relation between the integral of a function on the flow with the integral of a related function on the base. Let $f: Y \rightarrow \mathbb{R}$ be a continuous function. Define $\Delta_{f}: \Sigma^{+} \rightarrow \mathbb{R}$ by

$$
\Delta_{f}(x):=\int_{0}^{\tau(x)} f(x, t) \mathrm{d} t .
$$

Proposition 2.6 (Kac's Lemma). Let $f: Y \rightarrow \mathbb{R}$ be a continuous function and $\nu \in \mathcal{M}_{\Phi}$ an invariant measure that can be written as

$$
\nu=\frac{\mu \times m}{(\mu \times m)(Y)},
$$


where $\mu \in \mathcal{M}_{\sigma}$, then

$$
\int_{Y} f \mathrm{~d} \nu=\frac{\int_{\Sigma} \Delta_{f} \mathrm{~d} \mu}{\int_{\Sigma} \tau \mathrm{d} \mu}
$$

Propositions 2.5 and 2.6 together with the relation between the spaces of invariant measures for the flow and for the shift established by Ambrose and Kakutani (see subsection 2.3) allow us to study thermodynamic formalism for the flow by means of the corresponding one on the base.

2.6. Thermodynamic formalism for suspension flows. Let $\left(\Sigma^{+}, \sigma\right)$ be a topologically mixing countable Markov shift and $\tau: \Sigma^{+} \rightarrow \mathbb{R}$ a positive function bounded away from zero of summable variations. Denote by $(Y, \Phi)$ the suspension semi-flow over $\left(\Sigma^{+}, \sigma\right)$ with roof function $\tau$. Thermodynamic formalism has been studied in this context by several people with different degrees of generality: Savchenko [Sav], Barreira and Iommi [BI], Kempton [Ke] and Jaerisch, Kesseböhmer and Lamei [JKL]. Thermodynamic formalism for suspension flows where the base $\left(\Sigma^{+}, \sigma\right)$ is a sub-shift of finite type defined on a finite alphabet has been studied, for example, in [BR, PP]. The next result provides equivalent definitions for the pressure, $P_{\Phi}(\cdot)$, on the flow.

Theorem 2.7. Let $f: Y \rightarrow \mathbb{R}$ be a function such that $\Delta_{f}: \Sigma^{+} \rightarrow \mathbb{R}$ is of summable variations. Then the following equalities hold

$$
\begin{aligned}
P_{\Phi}(f) & :=\lim _{t \rightarrow \infty} \frac{1}{t} \log \left(\sum_{\varphi_{s}(x, 0)=(x, 0), 0<s \leqslant t} \exp \left(\int_{0}^{s} f\left(\varphi_{k}(x, 0)\right) \mathrm{d} k\right) \chi_{C_{i_{0}}}(x)\right) \\
& =\inf \left\{t \in \mathbb{R}: P_{\sigma}\left(\Delta_{f}-t \tau\right) \leqslant 0\right\}=\sup \left\{t \in \mathbb{R}: P_{\sigma}\left(\Delta_{f}-t \tau\right) \geqslant 0\right\} \\
& =\sup \left\{P_{\Phi \mid K}(f): K \in \mathcal{K}(\Phi)\right\},
\end{aligned}
$$

where $\mathcal{K}(\Phi)$ denotes the space of compact $\Phi$-invariant sets.

In particular, the topological entropy of the flow is the unique number $h_{t o p}(\Phi)$ satisfying

$$
h_{\text {top }}(\Phi)=\inf \{t \in \mathbb{R}: P(-t \tau) \leqslant 0\} .
$$

Note that in this setting the Variational Principle also holds (see [BI, JKL, Ke, Sav]).

Theorem 2.8 (Variational Principle). Let $f: Y \rightarrow \mathbb{R}$ be a function such that $\Delta_{f}: \Sigma^{+} \rightarrow \mathbb{R}$ is of summable variations. Then

$$
P_{\Phi}(f)=\sup \left\{h_{\nu}(\Phi)+\int_{Y} f \mathrm{~d} \nu: \nu \in \mathcal{M}_{\Phi} \text { and }-\int_{Y} f \mathrm{~d} \nu<\infty\right\} .
$$

A measure $\nu \in \mathcal{M}_{\Phi}$ is called an equilibrium measure for $f$ if

$$
P_{\Phi}(f)=h_{\nu}(\Phi)+\int f \mathrm{~d} \nu
$$

It was proved in [IJT, Theorem 3.5] that potentials $f$ for which $\Delta_{f}$ is locally Hölder have at most one equilibrium measure. Moreover, the following result (see [BI, Theorem 4]) characterises functions having equilibrium measures. 
Theorem 2.9. Let $f: Y \rightarrow \mathbb{R}$ be a continuous function such that $\Delta_{f}$ is of summable variations. Then there is an equilibrium measure $\nu_{f} \in \mathcal{M}_{\Phi}$ for $f$ if and only if we have that $P_{\sigma}\left(\Delta_{f}-P_{\Phi}(f) \tau\right)=0$ and there exists an equilibrium measure $\mu_{f} \in \mathcal{M}_{\sigma}$ for $\Delta_{f}-P_{\Phi}(f) \tau$ such that $\int \tau d \mu_{f}<\infty$.

Remark 2.10. We stress that the situation is more complicated when $\tau$ is not assumed to be bounded away from zero. For results in that setting see [IJT].

\section{ENTROPY AND ESCAPE OF MASS}

Over the last few years there has been interest, partially motivated for its connections with number theory, in studying the relation between entropy and the escape of mass of sequences of invariant measures for diagonal flows on homogenous spaces (see [EKP, ELMV, KLKM]). Some remarkable results have been obtained bounding the amount of mass that an invariant measure can give to an unbounded part of the domain (a cusp) in terms of the entropy of the measure (see for example [EKP, Theorem A] or [KLKM, Theorem 1.3]). The purpose of this section is to prove similar results in the context of suspension flows defined over countable Markov shifts. As we will see, the proofs in this setting suggest a geometrical interpretation that we pursue in section 5 .

Let $\left(\Sigma^{+}, \sigma\right)$ be a topologically mixing countable Markov shift of infinite topological entropy and $\tau: \Sigma^{+} \rightarrow \mathbb{R}^{+}$a potential of summable variations bounded away from zero. Denote by $(Y, \Phi)$ the associated suspension flow, which we assume to have finite topological entropy. Note that since $\left(\Sigma^{+}, \sigma\right)$ has infinite entropy and $\tau$ is non-negative, the entropy $h_{\text {top }}(\Phi)$ of the flow satisfies $P_{\sigma}(-h(\Phi) \tau) \leqslant 0$ (see equation (5)). Therefore, there exists a real number $s_{\infty} \in\left(0, h_{\text {top }}(\Phi)\right]$ such that

$$
P_{\sigma}(-t \tau)= \begin{cases}\text { infinite } & \text { if } t<s_{\infty} \\ \text { finite } & \text { if } t>s_{\infty}\end{cases}
$$

As it turns out the number $s_{\infty}$ will play a crucial role in our work.

For geodesic flows defined in non-compact manifolds there are vectors that escape through the cusps, they do not exhibit any recurrence property. That phenomena is impossible in the symbolic setting, every point will return to the base after some time. The following definition describes the set of points that escape on average (compare with an analogous definition given in [KLKM]).

Definition 3.1. We say that a point $(x, t) \in Y$ escapes on average if

$$
\lim _{n \rightarrow \infty} \frac{1}{n} \sum_{i=0}^{n-1} \tau\left(\sigma^{i} x\right)=\infty .
$$

We denote the set of all points which escape on average by $\mathcal{E}_{\mathcal{A}}(\tau)$.

Remark 3.2. Note that if $\nu \in \mathcal{M}_{\Phi}$ is ergodic and $\nu=(\mu \times m) /(\mu \times m)(Y)$ with $\mu \in \mathcal{M}_{\sigma}$ then Birkhoff's theorem implies that

$$
\lim _{n \rightarrow \infty} \frac{1}{n} \sum_{i=0}^{n-1} \tau\left(\sigma^{i} x\right)=\int \tau d \mu .
$$

Thus, no measure in $\mathcal{M}_{\Phi}$ is supported on $\mathcal{E}_{\mathcal{A}}(\tau)$. We can, however, describe the dynamics of the set $\mathcal{E}_{\mathcal{A}}(\tau)$ studying sequences of measures $\nu_{n} \in \mathcal{M}_{\Phi}$ such that the 
associated measures $\mu_{n} \in \mathcal{M}_{\sigma}$ satisfy

$$
\lim _{n \rightarrow \infty} \int \tau d \mu_{n}=\infty .
$$

In our first result we show that a measure of sufficiently large entropy can not give too much weight to the set of points for which the return time to the base is very high. More precisely,

Theorem 3.3. Let $c \in\left(s_{\infty}, h_{\text {top }}(\Phi)\right)$. There exists a constant $C>0$ such that for every $\nu \in \mathcal{M}_{\Phi}$ with $h_{\nu}(\Phi) \geqslant c$, we have that

$$
\int \tau d \mu \leqslant C
$$

Proof. Let $\nu \in \mathcal{M}_{\Phi}$ with $h_{\nu}(\Phi)=c$ and let $\mu \in \mathcal{M}_{\sigma}$ be the invariant measure satisfying $\nu=(\mu \times m) /((\mu \times m)(Y))$. By the Abramov formula we have

$$
h_{\mu}(\sigma)-c \int \tau d \mu=0 .
$$

We will consider the straight line $L(t):=h_{\mu}(\sigma)-t \int \tau d \mu$. Note that $L(c)=0$ and $L(0)=h_{\mu}(\sigma)$. Let $s \in\left(s_{\infty}, c\right)$. Note that $P_{\sigma}(-s \tau)<\infty$ and by the variational principle $L(s) \leqslant P_{\sigma}(-s \tau)$. This remark readily implies a bound on the slope of $L(t)$. Indeed,

$$
\int \tau d \mu \leqslant \frac{P_{\sigma}(-s \tau)}{c-s} .
$$

Thus the constant $C=P_{\sigma}(-s \tau) /(c-s)$ satisfies the theorem. In order to obtain the best possible constant we have to compute the infimum of the function defined for $s \in\left(s_{\infty}, c\right)$ by

$$
s \mapsto \frac{P_{\sigma}(-s \tau)}{c-s} .
$$

Remark 3.4. We stress that the constant $C$ in Theorem 3.3 depends only on the entropy bound $c$ and not on the measure $\nu$.

Remark 3.5. An implicit assumption in Theorem 3.3 is that $s_{\infty}<h_{\text {top }}(\Phi)$. In Section 4 we will see that in the geometrical context of geodesic flows this assumption has a very natural interpretation. Indeed, it will be shown to be equivalent to the parabolic gap property (see [DP, Section III] or Definition 4.13 for precise definitions).

Corollary 3.6. If $\left(\Sigma^{+}, \sigma\right)$ is a Markov shift defined countable alphabet satisfying the BIP condition then the best possible constant $C \in \mathbb{R}$ in Theorem 3.3 is given by

$$
C=\frac{P_{\sigma}\left(-s_{m} \tau\right)}{c-s_{m}}
$$

where $s_{m} \in \mathbb{R}$ is such that the equilibrium measure $\mu_{s_{m}}$ for $-s_{m} \tau$ satisfies

$$
c=\frac{h_{\mu_{s_{m}}}(\sigma)}{\int \tau d \mu_{s_{m}}} .
$$


Proof. Since the system satisfies BIP condition the function $P_{\sigma}(-s \tau)$, when finite, is differentiable (see Theorem 2.3). Moreover, its derivative is given by (see [Sa3, Theorem 6.5]),

$$
\left.\frac{d}{d s} P_{\sigma}(-s \tau)\right|_{s=s_{m}}=-\int \tau d \mu_{s_{m}}
$$

where $\mu_{s_{m}}$ is the (unique) equilibrium measure for $-s_{m} \tau$. The critical points of the function $s \mapsto \frac{P_{\sigma}(-s \tau)}{c-s}$ are those which satisfy

$$
(c-s) P_{\sigma}^{\prime}(-s \tau)+P_{\sigma}(-s \tau)=0 .
$$

Equivalently,

$$
-(c-s) \int \tau d \mu_{s}+h_{\mu_{s}}(\sigma)-s \int \tau d \mu_{s}=0 .
$$

Therefore, equation (6) is equivalent to

$$
c=\frac{h_{\mu_{s}}(\sigma)}{\int \tau d \mu_{s}} .
$$

In the next Theorem we prove that the entropy of the flow on $\mathcal{E}_{\mathcal{A}}(\tau)$ is bounded above by $s_{\infty}$ and that, under some additional assumptions, it is actually equal to it. This result could be thought of as a symbolic estimation for the entropy of a flow in a cusp. Theorem 3.7 is a refined version of a result first observed in [FJLR, Lemma 2.5] and used in the context of suspension flows in [IJ].

Theorem 3.7. Let $\left(\nu_{n}\right)_{n} \in \mathcal{M}_{\Phi}$ be an sequence of invariant probability measure for the flow of the form

$$
\nu_{n}=\frac{\mu_{n} \times m}{\left(\mu_{n} \times m\right)(Y)}
$$

where $\mu_{n} \in \mathcal{M}_{\sigma}$. If $\lim _{n \rightarrow \infty} \int \tau d \mu_{n}=\infty$ then

$$
\limsup _{n \rightarrow \infty} h_{\nu_{n}}(\Phi) \leqslant s_{\infty} .
$$

Moreover, if $s_{\infty}<h_{\text {top }}(\Phi)$, then there exists a sequence $\left(\nu_{n}\right)_{n} \in \mathcal{M}_{\Phi}$ such that $\lim _{n \rightarrow \infty} \int \tau d \mu_{n}=\infty$ and

$$
\lim _{n \rightarrow \infty} h_{\nu_{n}}(\Phi)=s_{\infty}
$$

Proof. Observe that the first claim is a direct consequence of Theorem 3.3. Let us construct now a sequence $\left(\nu_{n}\right)_{n} \in \mathcal{M}_{\Phi}$ with $\lim _{n \rightarrow \infty} \int \tau d \mu_{n}=\infty$ such that $\lim _{n \rightarrow \infty} h_{\nu_{n}}(\Phi)=s_{\infty}$. First note that it is a consequence of the approximation property of the pressure, that there exists a sequence of compact invariant sets $\left(K_{N}\right)_{N} \subset \Sigma$ such that $\lim _{N \rightarrow \infty} P_{K_{N}}(-t \tau)=P_{\sigma}(-t \tau)$. In particular, for every $n \in \mathbb{N}$ we have that

$$
\lim _{N \rightarrow \infty} P_{K_{N}}\left(-\left(s_{\infty}-1 / n\right) \tau\right)=\infty
$$

For the same reason, for any $n \in \mathbb{N}$ and $N \in \mathbb{N}$ we have that

$$
P_{K_{N}}\left(-\left(s_{\infty}+1 / n\right) \tau\right) \leqslant P_{\sigma}\left(-\left(s_{\infty}+1 / n\right) \tau\right)<\infty .
$$

Thus, given $n \in \mathbb{N}$ there exists $N \in \mathbb{N}$ such that

$$
n^{2}<\frac{P_{K_{N}}\left(-\left(s_{\infty}-1 / n\right) \tau\right)-P_{K_{N}}\left(-\left(s_{\infty}+1 / n\right) \tau\right)}{2 / n} .
$$


Since the function $t \mapsto P_{K_{N}}(-t \tau)$ is real analytic, by the mean value theorem there exists $t_{n} \in\left[s_{\infty}-1 / n, s_{\infty}+1 / n\right]$, such that $P_{K_{N}}^{\prime}\left(-t_{n} \tau\right)>n^{2}$. Denote by $\mu_{n}$ the equilibrium measure for $-t_{n} \tau$ in $K_{N}$. We have that

$$
n^{2}<P_{K_{N}}^{\prime}\left(-t_{n} \tau\right)=\int \tau d \mu_{n} .
$$

In particular the sequence $\left(\mu_{n}\right)_{n}$ satisfies

$$
\lim _{n \rightarrow \infty} \int \tau d \mu_{n}=\infty .
$$

Since $s_{\infty}<h_{t o p}(\Phi)$ we have that for $n \in \mathbb{N}$ large enough

$$
h_{\mu_{n}}(\sigma)-t_{n} \int \tau d \mu_{n}>0
$$

In particular

$$
t_{n}<\frac{h_{\mu_{n}}(\sigma)}{\int \tau d \mu_{n}} .
$$

Since $t_{n} \in\left(s_{\infty}-1 / n, s_{\infty}+1 / n\right)$ we have that

$$
s_{\infty}=\lim _{n \rightarrow \infty} t_{n} \leqslant \lim _{n \rightarrow \infty} \frac{h_{\mu_{n}}(\sigma)}{\int \tau d \mu_{n}}=\lim _{n \rightarrow \infty} h_{\nu_{n}}(\Phi) .
$$

But we already proved that the limit can not be larger than $s_{\infty}$, thus the result follows.

Corollary 3.8. If $s_{\infty}<h_{t o p}(\Phi)$, then the entropy at infinity $h_{\infty}(Y, \Phi)$ of the suspension flow satisfies

$$
h_{\infty}(Y, \Phi)=s_{\infty} .
$$

Proof. Let $\left(\nu_{n}\right)$ be a sequence of $\Phi$-invariant probability measures such that $\nu_{n} \rightarrow 0$. Since

$$
\int f d \nu_{n}=\frac{\int \Delta_{f} d \mu_{n}}{\int \tau d \mu_{n}}
$$

the only way to have $\int f d \nu_{n} \rightarrow 0$ for every continuous function $f \in C^{0}(Y)$ with compact support, is that

$$
\lim _{n \rightarrow \infty} \int \tau d \mu_{n}=\infty .
$$

Hence, Theorem 3.7 implies that $\limsup _{n \rightarrow \infty} h_{\nu_{n}}(\Phi) \leqslant s_{\infty}$. On the other hand, Theorem 3.7 also says that there exists a sequence $\left(\nu_{n}\right)$ of $\Phi$-invariant probability measures such that $\lim _{n \rightarrow \infty} \int \tau d \mu_{n}=\infty$ and

$$
\lim _{n \rightarrow \infty} h_{\nu_{n}}(\Phi)=s_{\infty}
$$

Again, since $\int f d \nu_{n}=\frac{\int \Delta_{f} d \mu_{n}}{\int \tau d \mu_{n}}$, we have that $\nu_{n} \rightarrow 0$, and the conclusion follows. 


\section{The geodesic flow on Extended Schottky Groups}

4.1. Some preliminaries in negative curvature. Let $X$ be a Hadamard manifold with pinched negative sectional curvature, that is a complete simply connected Riemannian manifold whose sectional curvature $K$ satisfies $-b^{2} \leqslant K \leqslant-1$ (for some fixed $b \geqslant 1$ ). Denote by $\partial X$ the boundary at infinity of $X$. Finally, denote by $d$ the Riemannian distance on $X$. A crucial object on the study of the dynamics of the geodesic flow is the Busemann function. Let $\xi \in \partial X$ and $x, y \in X$. For every geodesic ray $t \mapsto \xi_{t}$ pointing to $\xi$, the limit

$$
B_{\xi}(x, y):=\lim _{t \rightarrow \infty}\left[d\left(x, \xi_{t}\right)-d\left(y, \xi_{t}\right)\right]
$$

always exists, and is independent of the geodesic ray $\xi_{t}$ since $X$ has negative sectional curvature. The Busemann function $B: \partial X \times X^{2} \rightarrow \mathbb{R}$ is the continuous function defined as $B(\xi, x, y) \mapsto B_{\xi}(x, y)$. An (open) horoball based in $\xi$ and passing through $x$ is the set of $y \in X$ such that $B_{\xi}(x, y)>0$. In the hyperbolic case, when $X=\mathbb{D}$, an open horoball based in $\xi \in \mathbb{S}^{1}$ and passing trough $x \in \mathbb{D}$ is the interior of an euclidean circle containing $x$ and tangent to $\mathbb{S}^{1}$ at $\xi$.

Recall that every isometry of $X$ can be extended to a homeomorphism of $X \cup \partial X$. A very important property of the Busemann function is the following. If $\varphi: X \rightarrow X$ is an isometry of $X$, then for every $x, y \in X$, we have

$$
B_{\varphi \xi}(\varphi x, \varphi y)=B_{\xi}(x, y) .
$$

Let $o \in X$ be a reference point, which is often called the origin of $X$. The unit tangent bundle $T^{1} X$ of $X$ can be identified with $\partial^{2} X \times \mathbb{R}$, where $\partial^{2} X=$ $(\partial X \times \partial X) \backslash$ diagonal, via Hopf's coordinates. A vector $v \in T^{1} X$ is identified with $\left(v^{-}, v^{+}, B_{v^{+}}(o, \pi(v))\right)$, where $v^{-}$(resp. $\left.v^{+}\right)$is the negative (resp. positive) endpoint of the geodesic defined by $v$. Here $\pi: T^{1} X \rightarrow X$ is the natural projection of a vector to its base point. Observe that the geodesic flow $\left(g_{t}\right): T^{1} X \rightarrow T^{1} X$ acts by translation in the third coordinate of this identification.

Another crucial object in this setting is the Poincaré series. It is intimately related to the topological entropy of the geodesic flow.

Definition 4.1. Let $\Gamma$ be a discrete subgroup of isometries of $X$ and let $x \in X$. The Poincaré series $P_{\Gamma}(s, x)$ associated with $\Gamma$ is defined by

$$
P_{\Gamma}(s, x):=\sum_{g \in \Gamma} e^{-s d(x, g x)} .
$$

The critical exponent $\delta_{\Gamma}$ of $\Gamma$ is

$$
\delta_{\Gamma}:=\inf \left\{s \in \mathbb{R}: P_{\Gamma}(s, x)<\infty\right\} .
$$

The group $\Gamma$ is said to be of divergence type (resp. convergence type) if $P_{\Gamma}\left(\delta_{\Gamma}, x\right)=$ $\infty\left(\operatorname{resp} . P_{\Gamma}\left(\delta_{\Gamma}, x\right)<\infty\right)$.

Remark 4.2. As the sectional curvature of $X$ is bounded from below, the critical exponent is finite. Moreover, by the triangle inequality, it is independent of $x \in X$.

The isometries of $X$ are categorized in three types. Those fixing an unique point in $X$ called elliptic isometries. Those fixing an unique point in $\partial X$ called parabolic isometries. And finally, those fixing uniquely two points in $\partial X$ called hyperbolic isometries. For $g$ a non-elliptic isometry of $X$, denote by $\delta_{g}$ the critical exponent 
of the group $<g>$. If $g$ is hyperbolic it is fairly straightforward to see that $\delta_{g}=0$ and that the group $\langle g\rangle$ is of divergence type. If $g$ is parabolic, it was shown in [DP, Theorem III.1], that $\delta_{g} \geqslant \frac{1}{2}$.

Let $\Gamma$ be a discrete subgroup of isometries of $X$. Denote by $\Lambda$ the limit set of $\Gamma$, that is, the set $\Lambda=\overline{\Gamma \cdot o} \backslash \Gamma \cdot o$. The group $\Gamma$ is non-elementary if $\Lambda$ contains infinitely many elements. We recall the following fact proved in [DOP, Proposition $2]$.

Theorem 4.3. Let $\Gamma$ be a non-elementary discrete subgroup of isometries of a Hadamard manifold $X$. If $G$ is a divergence type subgroup of $\Gamma$ and its limit set is strictly contained in the limit set of $\Gamma$, then $\delta_{\Gamma}>\delta_{G}$.

In particular, if $\Gamma$ is a non-elementary discrete group of isometries and there is an element $g \in \Gamma$ such that $\langle g\rangle$ is of divergence type, then $\delta_{\Gamma}>\delta_{<g}$ (see also [DP, Theorem III.1]). Note that a non-elementary group always contains a hyperbolic isometry (in fact, infinitely many non-conjugate of them), hence a non-elementary group $\Gamma$ always satisfies $\delta_{\Gamma}>0$.

We end this subsection giving an important relation between the topological entropy of the geodesic flow and the critical exponent of a group. Let $X$ be a Hadamard manifold with pinched negative sectional curvature and let $\Gamma$ be a nonelementary free-torsion discrete subgroup of isometries of $X$. Denote by $\left(g_{t}\right)$ : $T^{1} X / \Gamma \rightarrow T^{1} X / \Gamma$ the geodesic flow on the unit tangent bundle of the quotient manifold $X / \Gamma$. Otal and Peigné [OP, Theorem 1] proved that, if the derivatives of the sectional curvature are uniformly bounded, then the topological entropy $h_{t o p}(g)$ of the geodesic flow equals the critical exponent of the Poincaré series of the group $\Gamma$, that is

$$
h_{t o p}(g)=\delta_{\Gamma}
$$

We stress the fact that the assumption on the derivatives of the sectional curvature is crucial in order to compute the topological entropy of the geodesic flow. This assumption implies the Hölder regularity of the strong unstable and stable foliations (see for instance [PPS, Theorem 7.3]), which is used in the proof of [OP, Theorem $1]$.

4.2. The symbolic model for extended Schottky groups. In this section we recall the definition of an extended Schottky group. To the best of our knowledge this definition has been introduced in 1998 by Dal'bo and Peigné [DP]. The basic idea is to extend the classical notion of Schottky groups to the context of manifolds where the non-wandering set of the geodesic flow is non-compact.

Let $X$ be a Hadamard manifold as in subsection 4.1. Let $N_{1}, N_{2}$ be non-negative integers such that $N_{1}+N_{2} \geqslant 2$ and $N_{2} \geqslant 1$. Consider $N_{1}$ hyperbolic isometries $h_{1}, \ldots, h_{N_{1}}$ and $N_{2}$ parabolic ones $p_{1}, \ldots, p_{N_{2}}$ satisfying the following conditions:

(C1) For $1 \leqslant i \leqslant N_{1}$ there exist in $\partial X$ a compact neighbourhood $C_{h_{i}}$ of the attracting point $\xi_{h_{i}}$ of $h_{i}$ and a compact neighbourhood $C_{h_{i}^{-1}}$ of the repelling point $\xi_{h_{i}^{+}}$of $h_{i}$, such that

$$
h_{i}\left(\partial X \backslash C_{h_{i}^{-1}}\right) \subset C_{h_{i}} .
$$


(C2) For $1 \leqslant i \leqslant N_{2}$ there exists in $\partial X$ a compact neighbourhood $C_{p_{i}}$ of the unique fixed point $\xi_{p_{i}}$ of $p_{i}$, such that

$$
\forall n \in \mathbb{Z}^{*} \quad p_{i}^{n}\left(\partial X \backslash C_{p_{i}}\right) \subset C_{p_{i}} .
$$

(C3) The $2 N_{1}+N_{2}$ neighbourhoods introduced in (1) and (2) are pairwise disjoint.

(C4) The elementary parabolic groups $<p_{i}>$, for $1 \leqslant i \leqslant N_{2}$, are of divergence type.

The group $\Gamma=<h_{1}, \ldots, h_{N_{1}}, p_{1}, \ldots, p_{N_{2}}>$ is a non-elementary free group which acts properly discontinuously and freely on $X$ (see [DP, Corollary II.2]). Such a group $\Gamma$ is called an extended Schottky group. Note that if $N_{2}=0$, that is the group $\Gamma$ only contains hyperbolic elements, then $\Gamma$ is a classical Schottky group and its geometric and dynamical properties are well understood. Indeed, in that case, the non-wandering set $\Omega \subset T^{1} X / \Gamma$ of the geodesic flow is compact, which implies that $\left.\left(g_{t}\right)\right|_{\Omega}$ is an Axiom A flow. If $N_{2} \geqslant 1$, then $X / \Gamma$ is a non-compact manifold and $\Omega$ is a non-compact subset of $T^{1} X / \Gamma$. Figure 1 below is an example of a Schottky group acting on the hyperbolic disk $\mathbb{D}$. It has two generators, one hyperbolic and the other parabolic.

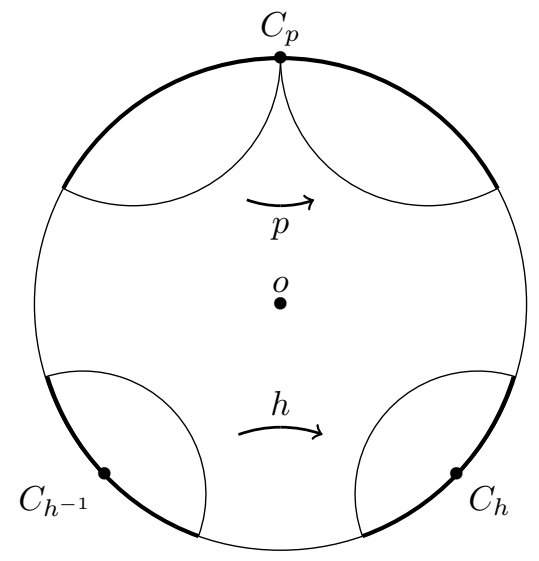

Figure 1. Schottky Group $\Gamma=<h, p>$.

Let $\mathcal{A}^{ \pm}=\left\{h_{1}^{ \pm 1}, \ldots, h_{N_{1}}^{ \pm 1}, p_{1}, \ldots, p_{N_{2}}\right\}$. We now define an extra hypothesis that we will use in large parts of this paper.

(C5) Let $a_{1}, a_{2} \in \mathcal{A}^{ \pm}$be such that $a_{1} \neq a_{2}^{ \pm 1}$. Then, there exists a point $o \in X$ such that for every $\xi \in C_{a_{1}}$, we have

$$
B_{\xi}\left(a_{2}^{ \pm 1} o, o\right)>0
$$

In other words, we will assume that every horoball based in $C_{a_{1}}$ and passing through $a_{2}^{ \pm 1} o$ contains the origin in his interior (see for instance Figure 2). This condition is not very restrictive as we will see in Proposition 4.5. 


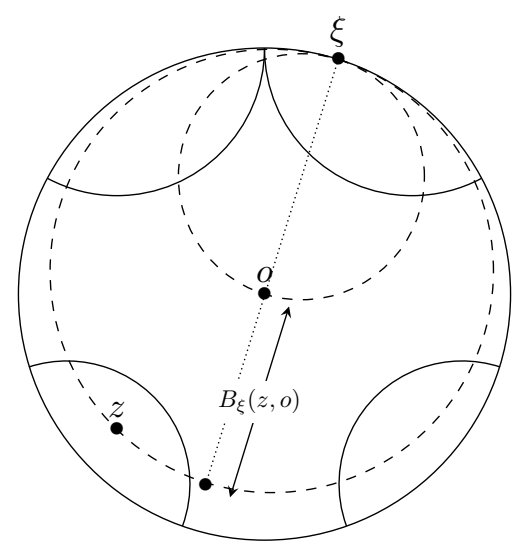

Figure 2 .

For $a \in \mathcal{A}^{ \pm}$denote by $U_{a}$ the convex hull in $X \cup \partial X$ of the set $C_{a}$.

Lemma 4.4. Let $X$ be a Hadamard manifold with pinched negative sectional curvature and let $\Gamma$ be an extended Schottky group. Fix $o \in X$. Then, there exists an universal constant $C>0$ (depending only on the generators of $\Gamma$ and the fixed point o) such that for every $a_{1}, a_{2} \in \mathcal{A}^{ \pm}$satisfying $a_{1} \neq a_{2}^{ \pm 1}$, and for every $x \in U_{a_{1}}$ and $y \in U_{a_{2}}$, we have

$$
d(x, y) \geqslant d(x, o)+d(y, o)-C .
$$

Proof. Since $C_{a_{1}}$ and $C_{a_{2}}$ are disjoint, for every $a_{1}, a_{2} \in \mathcal{A}^{ \pm}$satisfying $a_{1} \neq a_{2}^{ \pm 1}$, the same happens for the sets $U_{a_{1}}$ and $U_{a_{2}}$. Let $x \in U_{a_{1}}$ and $y \in U_{a_{2}}$. The geodesic segments $[o, x]$ and $[o, y]$ form an angle uniformly bounded below, hence $d(x, y) \geqslant d(x, o)+d(y, o)-C$ for an universal constant $C>0$.

Proposition 4.5. Let $X$ be a Hadamard manifold with pinched negative sectional curvature and $\Gamma=<h_{1}, \ldots, h_{N_{1}}, p_{1}, \ldots, p_{N_{2}}>$ an extended Schottky group. Then, for every $o \in X$ there exists an integer $N \geqslant 1$ such that the group defined by $<h_{1}^{N}, \ldots, h_{N_{1}}^{N}, p_{1}^{N}, \ldots, p_{N_{2}}^{N}>$ satisfies the Condition (C5).

Proof. Let $a_{1}, a_{2} \in \mathcal{A}^{ \pm}$and $\xi \in C_{a_{1}}$. Denote by $U_{a_{2}^{n}}$ the convex hull in $X \cup \partial X$ of the set $C_{a_{2}^{n}}$, for $n \geqslant 1$. Let $z_{n} \in U_{a_{2}^{n}}$ be such that $B_{\xi}\left(z_{n}, o\right)$ attains its minimum. Since $B$ is a continuous function and $C_{a_{1}}$ is a compact set, it is sufficient to prove that $B_{\xi}\left(z_{n}, o\right)>0$ for all $n$ large enough. Consider $\left(\xi_{t}\right)=\left[o, \xi_{t}\right)$ the geodesic ray starting in $o$ and pointing to $\xi$. Observe that there exists $T>0$ such that for every $t \geqslant T$, we have that $\xi_{t}$ belongs to $U_{a_{1}}$. Since $C_{a_{1}}$ and $C_{a_{2}}$ are disjoint, by Lemma 4.4 there exists a universal constant $C>0$ such that $d\left(z_{n}, \xi_{t}\right) \geqslant d\left(\xi_{t}, o\right)+d\left(z_{n}, o\right)-C$, for every $t \geqslant T$. Therefore,

$$
\begin{aligned}
B_{\xi}\left(z_{n}, o\right) & =\lim _{t \rightarrow+\infty} d\left(z_{n}, \xi_{t}\right)-d\left(\xi_{t}, o\right) \\
& \geqslant \lim _{t \rightarrow+\infty} d\left(\xi_{t}, o\right)+d\left(z_{n}, o\right)-C-d\left(\xi_{t}, o\right) \\
& =d\left(z_{n}, o\right)-C .
\end{aligned}
$$

Since $d\left(z_{n}, o\right) \rightarrow \infty$ as $n \rightarrow \infty$, there exists a $N \geqslant 1$ such that $d\left(z_{n}, o\right)>C$ for every $n \geqslant N$. In particular, we have $B_{\xi}\left(z_{n}, o\right)>0$ for every $n \geqslant N$ and the conclusion follows. 
In [DP] the authors proved that there exists a $\left(g_{t}\right)$-invariant subset $\Omega_{0}$ of $T^{1} X / \Gamma$, contained in the non-wandering set of $\left(g_{t}\right)$, such that $\left.\left(g_{t}\right)\right|_{\Omega_{0}}$ is topologically conjugated to a suspension flow over a countable Markov shift $(\Sigma, \sigma)$. The Theorem below summarizes their construction together with some dynamical properties.

Theorem 4.6. Let $X$ be a Hadamard manifold with pinched negative sectional curvature and let $\Gamma$ be an extended Schottky group. Suppose that $\Gamma$ satisfies Condition (C5). Then, there exists a $\left(g_{t}\right)$-invariant subset $\Omega_{0}$ of $T^{1} X / \Gamma$, a countable Markov shift $(\Sigma, \sigma)$ and a function $\tau: \Sigma \rightarrow \mathbb{R}$, such that

(1) the function $\tau$ is locally Hölder and is bounded away from zero,

(2) the geodesic flow $\left.\left(g_{t}\right)\right|_{\Omega_{0}}$ over $\Omega_{0}$ is topologically conjugated to the suspension flow over $\Sigma$ with roof function $\tau$,

(3) the Markov shift $(\Sigma, \sigma)$ satisfies the BIP condition,

(4) if $N_{1}+N_{2} \geqslant 3$, then $(\Sigma, \sigma)$ is topologically mixing.

Proof. Let $\mathcal{A}=\left\{h_{1}, \ldots, h_{N_{1}}, p_{1}, \ldots, p_{N_{2}}\right\}$ and consider the symbolic space $\Sigma$ defined by

$$
\Sigma=\left\{\left(a_{i}^{m_{i}}\right)_{i \in \mathbb{Z}}: a_{i} \in \mathcal{A}, m_{i} \in \mathbb{Z} \text { and } a_{i+1} \neq a_{i} \forall i \in \mathbb{Z}\right\} .
$$

Note that the space $\Sigma$ is a sequence space defined on the countable alphabet $\left\{a_{i}^{m}\right.$ : $\left.a_{i} \in \mathcal{A}, m \in \mathbb{Z}\right\}$. Let $\Lambda^{0}$ be the limit set $\Lambda$ minus the $\Gamma$-orbit of the fixed points of the elements of $\mathcal{A}$. We denote by $\tilde{\Omega}_{0}$ the set of vectors in $T^{1} X$ identified with $\left(\Lambda^{0} \times \Lambda^{0} \backslash\right.$ diagonal $) \times \mathbb{R}$ via Hopf's coordinates. Finally, define $\Omega_{0}:=\tilde{\Omega}_{0} / \Gamma$, where the action of $\Gamma$ is given by

$$
\gamma \cdot\left(\xi^{-}, \xi^{+}, s\right)=\left(\gamma\left(\xi^{-}\right), \gamma\left(\xi^{+}\right), s-B_{\xi^{+}}\left(o, \gamma^{-1} 0\right)\right) .
$$

Observe that $\Omega_{0}$ is invariant by the geodesic flow.

Fix now $\xi_{0} \in \partial X \backslash \bigcup_{a \in \mathcal{A}} C_{a^{ \pm}}$, where $C_{a^{ \pm}}=C_{a} \cup C_{a^{-1}}$. Dal'bo and Peigné [DP, Property II.5] established the following coding property: for every $\xi \in \Lambda^{0}$ there exists an unique sequence $\omega(\xi)=\left(a_{i}^{m_{i}}\right)_{i \geqslant 1}$ with $a_{i} \in \mathcal{A}, m_{i} \in \mathbb{Z}^{*}$ and $a_{i+1} \neq a_{i}$ such that

$$
\lim _{k \rightarrow \infty} a_{1}^{m_{1}} \ldots a_{k}^{m_{k}} \xi_{0}=\xi
$$

For each $a \in \mathcal{A}$ define $\Lambda_{a \pm}^{0}=\Lambda^{0} \cap C_{a^{ \pm}}$and set $\partial^{2} \Lambda^{0}=\bigcup_{\substack{\alpha, \beta \in \mathcal{A} \\ \alpha \neq \beta}} \Lambda_{\alpha^{ \pm}}^{0} \times \Lambda_{\beta \pm}^{0}$. For any pair $\left(\xi^{-}, \xi^{+}\right) \in \partial^{2} \Lambda^{0}$, if $a^{m}$ is the first term of the sequence $\omega\left(\xi^{+}\right)$, define $\tilde{\tau}\left(\xi^{+}\right)=B_{\xi^{+}}\left(o, a^{m} o\right)$ and $\bar{T}\left(\xi^{-}, \xi^{+}\right)=\left(a^{-m} \xi^{-}, a^{-m} \xi^{+}\right)$. Define $\bar{T}_{\tau}$ by the formula

$$
\bar{T}_{\tau}\left(\xi^{-}, \xi^{+}, s\right)=\left(\bar{T}\left(\xi^{-}, \xi^{+}\right), s-\tilde{\tau}\left(\xi^{+}\right)\right) .
$$

Observe that $\bar{T}_{\tau}$ maps $\partial^{2} \Lambda^{0} \times \mathbb{R}$ to itself. The set $\Omega_{0}$ can be identified with the quotient $\partial^{2} \Lambda^{0} \times \mathbb{R} /<\bar{T}_{\tau}>$. In order to prove this claim, we first proceed to explain how $\bar{T}_{\tau}$ gives a suspension flow.

Let $\left(\xi^{-}, \xi^{+}\right) \in \partial^{2} \Lambda^{0}$. Suppose that $\omega\left(\xi^{+}\right)=\left(a_{i}^{m_{i}}\right)$ and let $\gamma_{n}$ be the element of $\Gamma$ defined as $\gamma_{n}=a_{1}^{m_{1}} \ldots a_{n}^{m_{n}}$ for $n \geqslant 1$ and $\gamma_{0}=I d$. The geodesic determined by $\left(\xi^{-}, \xi^{+}\right)$in $X$ intersects the horosphere based in $\xi^{+}$and passing through $\gamma_{n} O$ in only one point $x_{o, \xi^{-}, \xi^{+}}^{n}$. Denote by $\tilde{v}_{o, \xi^{-}, \xi^{+}}^{n}$ the vector in $T^{1} X$ based in $x_{o, \xi^{-}, \xi^{+}}^{n}$ and pointing to $\xi^{+}$(see Figure 3 below). Finally, denote by $v_{o, \xi^{-}, \xi^{+}}^{n}$ the projection of $\tilde{v}_{o, \xi^{-}, \xi^{+}}^{n}$ over $T^{1} X / \Gamma$. Set

$$
S=\left\{v_{o, \xi^{-}, \xi^{+}}^{0}:\left(\xi^{-}, \xi^{+}\right) \in \partial^{2} \Lambda^{0}\right\} \subset T^{1} X / \Gamma .
$$


Condition (C5) implies that the first return time of every $v_{o, \xi^{-}, \xi^{+}}^{0} \in S$ is given by $\tilde{\tau}\left(\xi^{+}\right)$(which is the distance between the horospheres based in $\xi^{+}$and passing through $o$ and $\gamma_{1} o$ ). Moreover, $g_{\tilde{\tau}\left(\xi^{+}\right)}\left(v_{o, \xi^{-}, \xi^{+}}^{0}\right)=v_{o, \xi^{-}, \xi^{+}}^{1}$ (see Figure 3).

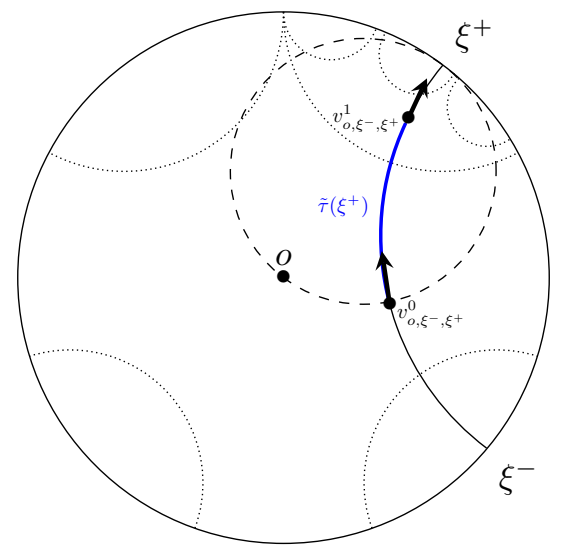

Figure 3. Cross section for $\Gamma=<h, p>$

More generally, for every $n \geqslant 0$ the first return time of $v_{o, \xi^{-}, \xi^{+}}^{n} \in S$ is given by $\tilde{\tau}\left(\gamma_{n}^{-1} \xi^{+}\right)$and

$$
g_{\tilde{\tau}\left(\gamma_{n}^{-1} \xi^{+}\right)}\left(v_{o, \xi^{-}, \xi^{+}}^{n}\right)=v_{o, \xi^{-}, \xi^{+}}^{n+1} .
$$

Note that for every vector $v \in \Omega_{0}$ there exists a time $t \in \mathbb{R}$ such that $g_{t} v$ belongs to $S$ (otherwise, a lift of $v$ to $T^{1} M$ would have its positive endpoint $v^{+}$in the $\Gamma$-orbit of a fixed point of an element in $\mathcal{A}$ ). This fact give us the identification.

The coding property implies that the set $\partial^{2} \Lambda^{0}$ is identified with $\Sigma$ by considering $\left(\xi^{-}, \xi^{+}\right)$as a bilateral sequence $\left(\omega^{*}\left(\xi^{-}\right), \omega\left(\xi^{+}\right)\right)$. If $\omega\left(\xi^{-}\right)=\left(b_{i}^{n_{i}}\right)_{i \geqslant 1}$, we define $\omega^{*}\left(\xi^{-}\right)$as the sequence $\left(\ldots, b_{2}^{-n_{2}}, b_{1}^{-n_{1}}\right)$, then $\left(\omega^{*}\left(\xi^{-}\right), \omega\left(\xi^{+}\right)\right)$represent the concatenated sequence. Let $\Sigma^{+}$be the one sided symbolic space obtained from $\Sigma$ by forgetting the negative time coordinates. We define the function $\tau: \Sigma^{+} \rightarrow \mathbb{R}$ as

$$
\tau(x)=\tau\left(\omega^{-1}(x)\right)=B_{\omega^{-1}(x)}\left(o, a^{m} o\right),
$$

where $w: \Lambda^{0} \rightarrow \Sigma$ is the coding function and $a^{m}$ the first symbol in $w^{-1}(x)$. We extend $\tau$ to $\Sigma$ by making it independent of the negative time coordinates. We therefore have, that the geodesic flow $\left(g_{t}\right)$ on $\Omega_{0}$ can be coded as the suspension flow on $Y=\{(x, t) \in \Sigma \times \mathbb{R}: 0 \leqslant t \leqslant \tau(x)\} / \sim$. This implies (2) in the conclusion of Theorem 4.6. Property (1) follows from Lemma 4.7 below.

Lemma 4.7. Under the hypothesis of Theorem 4.6, the function $\tau: \Sigma \rightarrow \mathbb{R}$ depends only on future coordinates, it is locally Hölder and it is bounded away from zero.

Proof. The fact that it depends on the future is by definition and the regularity property was established in [DP, Lemma VII.]. Let $x \in \Sigma^{+}$and $\xi=\omega^{-1}(x)$ the point in $\Lambda^{0}$ associated to $x$ by the coding property. The function $\tau$ satisfies

$$
\tau(x)=B_{\xi}\left(o, a^{m} o\right)=B_{a^{-m} \xi}\left(a^{-m} o, o\right) .
$$

The last term above is greater than 0 by Condition (C5). Indeed, note that $a^{-m} \xi=$ $\omega^{-1}(\sigma x) \notin C_{a^{ \pm}}$and $a^{-m} O$ is contained in the convex-hull of $C_{a^{ \pm}}$in $X \times \partial X$, 
hence Condition (C5) applies directly. Since the domains $C_{a}$, for $a \in \mathcal{A}^{ \pm}$, are compact and $B$ is continuous, there exists an uniform strictly positive lower bound for $B_{a^{-m} \xi}\left(a^{-m} o, o\right)$. Thus $\tau$ is bounded away from zero.

Lemma 4.8. Under the hypothesis of Theorem 4.6 the countable Markov shift $(\Sigma, \sigma)$ satisfies the BIP condition. Moreover, if $N_{1}+N_{2} \geqslant 3$, then the countable Markov shift $(\Sigma, \sigma)$ is topologically mixing.

Proof. It is not hard to see that the set $\mathcal{A}$ satisfies the required conditions in order for $(\Sigma, \sigma)$ to be BIP (see definition 2.2). Suppose now that $N_{1}+N_{2} \geqslant 3$. Recall that the Markov shift $(\Sigma, \sigma)$ is topologically mixing if for every $a, b \in\left\{a_{i}^{m}: a_{i} \in \mathcal{A}, m \in \mathbb{Z}\right\}$ there exists $N(a, b) \in \mathbb{N}$ such that for every $n>N(a, b)$ there exists an admissible word of length $n$ of the form $a i_{1} i_{2} \ldots i_{n-1} b$. The set of allowable sequences is given by

$$
\left\{\left(a_{i}^{m_{i}}\right)_{i \in \mathbb{Z}}: a_{i} \in \mathcal{A}, m_{i} \in \mathbb{Z} \text { and } a_{i+1} \neq a_{i} \forall i \in \mathbb{N}\right\} .
$$

Since $N_{1}+N_{2} \geqslant 3$ then given any pair of symbols in $\left\{a_{i}^{m}: a_{i} \in \mathcal{A}, m \in \mathbb{Z}\right\}$, say $a_{1}^{m_{1}}, a_{2}^{m_{2}}$ we can consider the symbol $a_{3} \notin\left\{a_{1}, a_{2}\right\}$. Hence the following words are admissible:

$$
a_{1}^{m_{1}} a_{3} a_{1} a_{3} \ldots a_{1} a_{2}^{m_{2}}, a_{1}^{m_{1}} a_{3} a_{1} a_{3} a_{1} \ldots a_{3} a_{2}^{m_{2}} .
$$

Thus, the system is topologically mixing.

Since Lemma 4.8 above shows the points (3) and (4), we have concluded the proof of Theorem 4.6.

Remark 4.9. Under the conditions $(C 5)$ and $N_{1}+N_{2} \geqslant 3$ we have proved that $(\Sigma, \sigma)$ is a topologically mixing countable Markov shift satisfying the BIP condition (Lemma 4.8) and that the roof function $\tau$ is locally Hölder and bounded away from zero (Lemma 4.7). Therefore, the associated suspension semi-flow $(Y, \Phi)$ can be studied with the techniques presented in Section 2.

So far we have proved that the geodesic flow restricted to the set $\Omega_{0}$ can be coded by a suspension flow over a countable Markov shift. We now describe, from the ergodic point of view, the geodesic flow in the complement $\left(T^{1} X / \Gamma\right) \backslash \Omega_{0}$ of $\Omega_{0}$. We denote by $\mathcal{M}_{\Omega_{0}}$ the space of $\left(g_{t}\right)$-invariant probability measures supported in the set where we have coding, in other words in $\partial^{2} \Lambda_{0} \times \mathbb{R} /<\bar{T}_{\tau}>$. We describe the difference between the space $\mathcal{M}_{\Omega_{0}}$ and the space $\mathcal{M}_{g}$ of all $\left(g_{t}\right)$-invariant probability measures. Recall that in $\Gamma$ there are hyperbolic isometries $h_{1}, \ldots, h_{N_{1}}$, each of which fixes a pair of points in $\partial X$. The geodesic connecting the fixed points of $h_{i}$ will descend to a closed geodesic in the quotient by $\Gamma$. We denote $\nu^{h_{i}}$ the probability measure equidistributed along such a geodesic.

Proposition 4.10. The set of ergodic measures in $\mathcal{M}_{g} \backslash \mathcal{M}_{\Omega_{0}}$ is finite, those are exactly the set $\left\{\nu^{h_{i}}: 1 \leqslant i \leqslant N_{1}\right\}$. Moreover, for every $\nu \in \mathcal{M}_{g} \backslash \mathcal{M}_{\Omega_{0}}$ we have $h_{\nu}(g)=0$.

Proof. Let $\nu \in \mathcal{M}_{g} \backslash \mathcal{M}_{\Omega_{0}}$ be an ergodic measure. Take $v$ a generic vector for $\nu$. Since a generic vector is recurrent, the orbit $g_{t} v$ does not goes to infinity, therefore $v^{+}$is not parabolic. Now consider the case when $v$ points toward a hyperbolic fixed point $z$. Let $\gamma: \mathbb{R} \rightarrow X$ be a geodesic flowing at positive time to $z$ with initial condition $\gamma^{\prime}(0)=v$ and let $\gamma_{i}$ be the geodesic connecting $z$ with the associated hyperbolic fixed point. By reparametrization we can assume $\gamma_{i}(+\infty)=z$ and that 
$\gamma_{i}(0)$ lies in the same horosphere centered at $z$ than $v$. By estimates in [HI] we have $d\left(\gamma_{i}(t), \gamma(t)\right) \rightarrow 0$ exponentially fast (here $d$ stands for hyperbolic distance, actually in [HI] the stronger exponential decay in the horospherical distance is obtained). Since the vectors along the geodesics are perpendicular to the horospheres centered at $z$ we have the desired geometric convergence in $T X$. Observe $\gamma_{i}$ descends to a periodic orbit in $T X / \Gamma$. This gives the convergence of $\gamma$ to the periodic orbit and Birkhoff ergodic theorem gives that the measure generated by such a geodesic is exactly one of $\nu^{h_{i}}$.

The fact that $h_{\nu}(g)=0$ for every $\nu \in \mathcal{M}_{g} \backslash \mathcal{M}_{\Omega_{0}}$ is a classical result for measures supported on periodic orbits.

We end up this section with a definition that includes the standing assumptions on the Schottky groups considered in most of our statements. The important point is that we will be in position to use Lemma 4.7, Lemma 4.8 and Theorem 4.12 below.

Definition 4.11. We say a extended Schottky group $\Gamma$ satisfies property $(\star)$ if conditions (C5) and $N_{1}+N_{2} \geqslant 3$ hold.

4.3. Geometric meaning of $s_{\infty}$. One of our main technical results is the following. Let $\tau$ be the roof function constructed in sub-section 4.2. In the next Theorem we give a geometrical characterisation of the value $s_{\infty}$ defined as the unique real number satisfying

$$
P_{\sigma}(-t \tau)= \begin{cases}\text { infinite } & \text { if } t<s_{\infty} \\ \text { finite } & \text { if } t>s_{\infty} .\end{cases}
$$

One of the key ingredients in this paper, and the important result of this section, is the relation between $s_{\infty}$ and the largest parabolic critical exponent. This relation will allow us to translate several results at the symbolic level into the geometrical one.

Theorem 4.12. Let $\Gamma$ be an extended Schottky group with property $(\star)$. Let $(\Sigma, \sigma)$ and $\tau: \Sigma \rightarrow \mathbb{R}$ be the base space and the roof function of the symbolic representation of the geodesic flow $\left(g_{t}\right)$ on $\Omega_{0}$. Then $s_{\infty}=\max \left\{\delta_{p_{i}}, 1 \leqslant i \leqslant N_{2}\right\}$.

Proof. We first show that $s_{\infty} \leqslant \max \left\{\delta_{p_{i}}, 1 \leqslant i \leqslant N_{2}\right\}$.

$$
\begin{aligned}
P_{\sigma}(-t \tau) & =\lim _{n \rightarrow \infty} \frac{1}{n+1} \log \sum_{x: \sigma^{n+1} x=x} \exp \left(\sum_{i=0}^{n}-t \tau\left(\sigma^{i} x\right)\right) \chi_{C_{h_{1}}}(x) \\
& =\lim _{n \rightarrow \infty} \frac{1}{n+1} \log \sum_{\xi=\overline{h_{1} x_{2} \ldots x_{n} x_{n+1}} \xi_{0}} \exp \left(\sum_{i=0}^{n}-t B_{\omega^{-1}\left(\sigma^{i} x\right)}\left(o, x_{i+1} o\right)\right) \\
& \geqslant \lim _{n \rightarrow \infty} \frac{1}{n+1} \log \sum_{\xi=\frac{h_{1} x_{2} \ldots x_{n+1}}{} \xi_{0}} \exp \left(\sum_{i=0}^{n}-t d\left(o, x_{i+1} o\right)\right)
\end{aligned}
$$

The last inequality follows from $d(x, y) \geqslant B_{\xi}(x, y)$. By removing words having $h_{1}^{m}$ (some $m$ ) in more places than just the first coordinate, we conclude that the argument of the function $\log$ in the limit above is greater than

$$
e^{-t d\left(o, h_{1} o\right)} \sum_{\left(c_{1}, \ldots, c_{n}\right) \in\left(\mathcal{A} \backslash h_{1}\right)_{*}^{n}} \sum_{\left(m_{1}, \ldots, m_{n}\right) \in \mathbb{Z}^{n}} \exp \left(\sum_{i=1}^{n}-t d\left(o, c_{i}^{m_{i}} o\right)\right),
$$


where $\left(\mathcal{A} \backslash h_{1}\right)_{*}^{n}$ represent the set of admissible words of length $n$ for the code, i.e. $c_{i} \neq c_{i+1}^{ \pm 1}$. Let $k \geqslant 1$. For all $0 \leqslant j \leqslant k-1$ and $1 \leqslant i \leqslant N_{1}+N_{2}-1$, define

$$
b_{i+j\left(N_{1}+N_{2}-1\right)}= \begin{cases}h_{i+1}, & \text { if } 1 \leqslant i \leqslant N_{1}-1 \\ p_{i+1-N_{1}}, & \text { if } N_{1} \leqslant i \leqslant N_{1}+N_{2}-1 .\end{cases}
$$

Consider $n+1=k\left(N_{1}+N_{2}-1\right)$. By restricting the above sum to words with $c_{i}=b_{i}$ for every $i=1, \ldots, n$, we can continue the sequence of inequalities above to get

$$
P_{\sigma}(-t \tau) \geqslant \sum_{m_{1}, \ldots, m_{n} \in \mathbb{Z}} \exp \left(\sum_{i=1}^{n}-t d\left(o, b_{i}^{m_{i}} o\right)\right),
$$

where the right-hand side is equal to

$$
\prod_{i=1}^{n} \sum_{m \in \mathbb{Z}} \exp \left(-t d\left(o, b_{i}^{m} o\right)\right) \text {. }
$$

By definition of the $b_{i}$ 's, the last term is equal to

$$
\left(\prod_{i=2}^{N_{1}} \sum_{m \in \mathbb{Z}} \exp \left(-t d\left(o, h_{i}^{m} o\right)\right)\right)^{k}\left(\prod_{i=1}^{N_{2}} \sum_{m \in \mathbb{Z}} \exp \left(-t d\left(o, p_{i}^{m} o\right)\right)\right)^{k} .
$$

Hence, it follows that

$$
\begin{aligned}
P_{\sigma}(-t \tau) & \geqslant \frac{1}{N_{1}+N_{2}} \log \left(\prod_{i=2}^{N_{1}} \sum_{m \in \mathbb{Z}} \exp \left(-t d\left(o, h_{i}^{m} o\right)\right)\right)\left(\prod_{i=1}^{N_{2}} \sum_{m \in \mathbb{Z}} \exp \left(-t d\left(o, p_{i}^{m} o\right)\right)\right) \\
& =\frac{1}{N_{1}+N_{2}} \log \prod_{a \in \mathcal{A} \backslash h_{1}} P_{<a>}(t, o) .
\end{aligned}
$$

In particular, if $t<\max \left\{\delta_{p_{i}}, 1 \leqslant i \leqslant N_{2}\right\}$ then $P_{\sigma}(-t \tau)=+\infty$. This shows that $s_{\infty} \geqslant \max \left\{\delta_{p_{i}}, 1 \leqslant i \leqslant N_{2}\right\}$.

We prove now the other inequality. Let $\left(\xi_{t}^{i}\right)$ be the geodesic ray $\left[o, \omega^{-1}\left(\sigma^{i+1} x\right)\right)$. Using (12), we have

$$
\begin{aligned}
\tau\left(\sigma^{i} x\right) & =B_{\omega^{-1}\left(\sigma^{i} x\right)}\left(o, x_{i} o\right) \\
& =B_{\omega^{-1}\left(\sigma^{i+1} x\right)}\left(x_{i}^{-1} o, o\right) \\
& =\lim _{t \rightarrow \infty} d\left(\xi_{t}^{i}, x_{i} o\right)-d\left(\xi_{t}^{i}, o\right) \\
& \geqslant\left[d\left(\xi_{t}^{i}, o\right)+d\left(o, x_{i} o\right)-C\right]-d\left(\xi_{t}^{i}, o\right) \\
& =d\left(o, x_{i} o\right)-C .
\end{aligned}
$$

Thus,

Therefore,

$$
\exp \left(-t \tau\left(\sigma^{i} x\right)\right) \leqslant \exp (t C) \exp \left(-t d\left(o, x_{i} o\right)\right)
$$

$$
\begin{aligned}
P_{\sigma}(-t \tau) & \leqslant \lim _{n \rightarrow \infty} \frac{1}{n} \log \sum_{a_{1}, \ldots, a_{n}} \sum_{m_{1}, \ldots, m_{n}} \prod_{i=1}^{n} \exp (t C) \exp \left(-t d\left(o, a_{i}^{m_{i}} o\right)\right) \\
& =\log \left(C^{t} \prod_{a \in \mathcal{A}} P_{<a>}(t, o)\right)
\end{aligned}
$$


In particular, the pressure $P_{\sigma}(-t \tau)$ is finite for every $t>\max \left\{\delta_{p_{i}}, 1 \leqslant i \leqslant N_{2}\right\}$.

Denote by $\delta_{p, \max }:=\max \left\{\delta_{p_{i}}, 1 \leqslant i \leqslant N_{2}\right\}$. The simplest example to consider is a real hyperbolic space $X$. In this case $\delta_{<p_{i}>}=1 / 2$ for any $i \in\left\{1, \ldots, N_{2}\right\}$. In particular $\delta_{p, \max }=1 / 2$. More generally, if we replace hyperbolic space by a manifold of constant negative curvature equal to $-b^{2}$ then $\delta_{<p>}=b / 2$. In the case of non-constant curvature some bounds are known, indeed if the curvature is bounded above by $-a^{2}$ then $\delta_{<p>} \geqslant a / 2$ (see [DOP]).

Recall that at a symbolic level we have $h_{t o p}(\Phi)=\inf \left\{t: P_{\sigma}(-t \tau) \leqslant 0\right\}$. In particular, if the derivatives of the sectional curvature are uniformly bounded, then Theorem 4.6, Proposition 4.10 and equality (11) imply

$$
h_{\text {top }}(g)=\delta_{\Gamma}=h_{\text {top }}(\Phi) \text {. }
$$

The existence of a measure of maximal entropy for the flow $\left(g_{t}\right)$ is related to convergence properties of the Poincaré series at the critical exponent. Indeed, using the construction of Patterson and Sullivan ([Pa], [Su]) of a $\Gamma$-invariant measure on $\partial^{2} X$, it is possible to construct a measure on $T^{1} X$ which is invariant under the action of $\Gamma$ and the geodesic flow. This measure induces a $\left(g_{t}\right)$-invariant measure on $T^{1} X / \Gamma$ called the Bowen-Margulis measure. It turns out that, if the group $\Gamma$ is of convergence type then the Bowen-Margulis measure is infinite and dissipative. Hence the geodesic flow does not have a measure of maximal entropy. On the other hand, if the group $\Gamma$ is of divergence type then the Bowen-Margulis measure is ergodic and conservative. If finite, it is the measure of maximal entropy.

It is, therefore, of interest to determine conditions that will ensure that the group is of divergence type and that the Bowen-Margulis measure is finite. It is along these lines that Dal'bo, Otal and Peigné [DOP] introduced the following:

Definition 4.13. A geometrically finite group $\Gamma$ satisfies the parabolic gap condition (PGC) if its critical exponent $\delta_{\Gamma}$ is strictly greater than the one of each of its parabolic subgroups.

It was shown in [DOP, Theorem A] that if a group satisfies the PGC-condition then the group is divergent and the measure of Bowen-Margulis is finite [DOP, Theorem B]. In particular it has a measure of maximal entropy. Note that a divergent group in the case of constant negative curvature satisfies the PGC-property.

In our context, an extended Schottky group is a geometrically finite group such that all the parabolic subgroups have rank 1. Moreover, by Condition (C4) and Theorem 4.3, it satisfies the PGC-condition. Thus, the following property is a direct consequence of Theorem 4.12, Theorem 4.3 and (13).

Proposition 4.14. Let $X$ be a Hadamard manifold with pinched negative sectional curvature and let $\Gamma$ be an extended Schottky group. Assume that the derivatives of the sectional curvature are uniformly bounded. If $(Y, \Phi)$ is the symbolic representation of the geodesic flow on $T^{1} X / \Gamma$, then $s_{\infty}<h_{\text {top }}(\Phi)$.

\section{ESCAPE OF MASS FOR GEODESIC FLOWS}

This section contains our main results relating the escape of mass of a sequence of invariant probability measures for a class of geodesic flows defined over noncompact manifolds. We prove that there is a uniform bound, depending only in the entropy of a measure, for the amount of mass a measure can give to the cusps. We also characterise the amount of entropy that the cusp can have. These results 
are similar in spirit to those obtained in [EKP, ELMV, KLKM] for other types of flows.

Theorem 5.1. Let $X$ be a Hadamard manifold with pinched negative sectional curvature and let $\Gamma$ be an extended Schottky group of isometries of $X$ with property $(\star)$. Assume that the derivatives of the sectional curvature are uniformly bounded. Then, for every $c>\delta_{p, \max }$ there exists a constant $M=M(c)>0$ such that for every $\nu \in \mathcal{M}_{\Omega_{0}}$ with $h_{\nu}(g) \geqslant c$, we have

$$
\int \tau d \mu \leqslant M
$$

where $\nu$ has a symbolic representation as $(\mu \times m) /((\mu \times m)(Y))$. Moreover, the value $\delta_{p, \text { max }}$ is optimal in the following sense: there exists a sequence $\nu_{n} \in \mathcal{M}_{\Omega_{0}}$ of $g$-invariant probability measures such that $\lim _{n \rightarrow \infty} \int \tau d \mu_{n}=\infty$ and

$$
\lim _{n \rightarrow \infty} h_{\nu_{n}}(g)=\delta_{p, \max }
$$

Proof. This is a direct consequence of Theorems 3.3 and 3.7 using the symbolic model for the geodesic flow on $T^{1} X / \Gamma$.

The following corollary is just an equivalence of the first conclusion in Theorem 5.1 (see also Theorem 3.7).

Corollary 5.2. Assume $X$ and $\Gamma$ as in Theorem 5.1. If $\nu_{n} \in \mathcal{M}_{\Omega_{0}}$ is a sequence of $\left(g_{t}\right)$-invariant probability measures such that $\lim _{n \rightarrow \infty} \int \tau d \mu_{n}=\infty$, then

$$
\limsup _{n \rightarrow \infty} h_{\nu_{n}}(g) \leqslant \delta_{p, \max } .
$$

We are now in position to prove the main result about escape of mass.

Theorem (1.2). Let $X$ be a Hadamard manifold with pinched negative sectional curvature and let $\Gamma$ be an extended Schottky group of isometries of $X$ with property $(\star)$. Assume that the derivatives of the sectional curvature are uniformly bounded. Then, for every $c>\delta_{p, \max }$ there exists a constant $m=m(c)>0$, with the following property: If $\left(\nu_{n}\right)$ is a sequence of ergodic $\left(g_{t}\right)$-invariant probability measures on $T^{1} X / \Gamma$ satisfying $h_{\nu_{n}}(g) \geqslant c$, then for every vague limit $\nu_{n} \rightarrow \nu$, we have

$$
\nu\left(T^{1} X / \Gamma\right) \geqslant m \text {. }
$$

In particular if $\nu_{n} \rightarrow 0$ then $\lim \sup h_{\nu_{n}}(g) \leqslant \delta_{p, \max }$. Moreover, the value $\delta_{p, \max }$ is optimal in the following sense: there exists a sequence $\left(\nu_{n}\right)$ of $\left(g_{t}\right)$-invariant probability measures on $T^{1} X / \Gamma$ such that $h_{\nu_{n}}(g) \rightarrow \delta_{p, \max }$ and $\nu_{n} \rightarrow 0$.

Proof. Since every ergodic measure in $\mathcal{M}_{g} \backslash \mathcal{M}_{\Omega_{0}}$ has zero entropy, we can suppose that $\nu_{n}$ belongs to $\mathcal{M}_{\Omega_{0}}$ for every $n \in \mathbb{N}$. Observe now that the cross-section $S \subset T^{1} X / \Gamma$ defined in the proof of Theorem 4.6 is bounded. Hence, using the identification $\Psi: \Omega_{0} \rightarrow Y$ given by Theorem 4.6 and fixing $0<r \leqslant \inf _{x \in \Sigma} \tau(x)$, there exists a compact set $K_{r} \subset T^{1} X / \Gamma$ such that

$$
\Sigma \times[0, r] / \sim \subset \Psi\left(K_{r}\right) .
$$

Let $\mu_{n}$ be the probability measure on $\Sigma$ associated to the symbolic representation of $\nu_{n}$. By Theorem 5.1, we have

$$
\int \tau d \mu_{n} \leqslant M
$$


Hence,

$$
\begin{aligned}
\nu_{n}\left(K_{r}\right) & =\Psi_{*} \nu_{n}\left(\Psi\left(K_{r}\right)\right) \geqslant \Psi_{*} \nu_{n}(\Sigma \times[0, r] / \sim) \\
& =\frac{\int_{\Sigma} \int_{0}^{r} d t d \mu_{n}}{\int \tau d \mu_{n}} \\
& \geqslant \frac{r}{M} .
\end{aligned}
$$

In other words, every vague limit $\nu$ of the sequence of ergodic probability measures $\left(\nu_{n}\right)_{n}$ satisfies $\nu\left(K_{r}\right) \geqslant r / M$. In particular, we obtain $\nu\left(T^{1} X / \Gamma\right) \geqslant r / M$. By setting $m=\inf _{x \in \Sigma} \tau(x) / M$, the conclusion follows.

Before giving the proof of the optimality of $\delta_{p, \max }$, we need the following result.

Proposition 5.3. Let $\Gamma$ be an extended Schottky group of isometries of $X$. Let $p \in \mathcal{A}$ be a parabolic isometry. We can choose a hyperbolic isometry $h \in \Gamma$ for which the groups $\Gamma_{n}=<p, h^{n}>$ satisfy the following conditions:

(1) The group $\Gamma_{n}$ is of divergence type for every $n \geqslant 1$,

(2) The sequence $\left(\delta_{\Gamma_{n}}\right)_{n}$ of critical exponents satisfy $\delta_{\Gamma_{n}} \rightarrow \delta_{\mathcal{P}}$ as $n$ goes to $\infty$,

(3) The following limit holds

$$
\lim _{n \rightarrow \infty} \frac{n}{\sum_{\gamma \in \mathcal{P}} e^{-\delta_{\Gamma_{n}} d(x, \gamma x)}}=0 .
$$

Proof. The proof is based on that of [DOP, Theorem C]. Let $G$ be a group, we will use the notation $G^{*}$ for $G \backslash\{i d\}$. Define $\mathcal{P}=<p>$ and take $U_{\mathcal{P}} \subset X \cup \partial X$ a connected compact neighbourhood of the fixed point $\xi_{p}$ of $p$ such that for every $m \in \mathbb{Z}^{*}$ we have $p^{m}\left(\partial X \backslash U_{\mathcal{P}}\right) \subset U_{\mathcal{P}}$. We could take $U_{\mathcal{P}}$ so that $U_{\mathcal{P}} \cap \partial X$ is a fundamental domain for the action of $\mathcal{P}$ in $\partial X \backslash\left\{\xi_{p}\right\}$. Because $\Gamma$ is non-elementary and $\Lambda_{\Gamma}$ is not contained in $U_{\mathcal{P}}$, it is possible to choose $h \in \Gamma$ a hyperbolic isometry of $X$ such that its two fixed points $\xi_{h^{-}}, \xi_{h}$ do not lie in $U_{\mathcal{P}}$. We have used the fact that pair of points fixed by a hyperbolic isometry is dense in $\Lambda \times \Lambda$. Fix $x \in X$ over the axis of $h$. Since $\Gamma$ is an extended Schottky group, for every $k \in \mathbb{N}$ the elements $p$ and $h^{k}$ are in Schottky position. In particular, for $H_{k}=<h^{k}>$ we can find a compact subset $U_{H_{k}} \subset X \cup \partial X$ satisfying the following three conditions

(1) $H^{*}\left(\partial X \backslash U_{H_{k}}\right) \subset U_{H_{k}}$.

(2) $U_{H_{k}} \cap U_{\mathcal{P}}=\varnothing$.

(3) $x \notin U_{H_{k}} \cup U_{\mathcal{P}}$.

Since $\mathcal{P}$ and $U_{H_{k}}$ are in Schottky position it is a consequence of the Ping Pong Lemma that the group generated by them is a free product. By the same argument to that one of Lemma 4.4, there is a positive constant $C \in \mathbb{R}$ such that for every $y \in U_{H_{k}}$ and $z \in U_{\mathcal{P}}$, we have

$$
d(y, z) \geqslant d(x, y)+d(x, z)-C .
$$

Applying inequality (14) and the inclusion properties described above we obtain

$$
d\left(x, p^{m_{1}} h^{k n_{1}} . . p^{m_{j}} h^{k n_{j}} x\right) \geqslant \sum_{i} d\left(x, p^{m_{i}} x\right)+\sum_{i} d\left(x, h^{k n_{i}} x\right)-2 k C,
$$

where $m_{i} \in \mathbb{Z}^{*}$. As remarked in $[\mathrm{DOP}]$ the sum

$$
\tilde{P}(s)=\sum_{j \geqslant 1} \sum_{n_{i}, m_{i} \in \mathbb{Z}^{*}} \exp \left(-s d\left(x, p^{m_{1}} h^{k n_{1}} \ldots p^{m_{j}} h^{k n_{j}} x\right)\right),
$$


is comparable with the Poincaré series of $\Gamma_{k}$. Indeed, since $h$ is hyperbolic both have the same critical exponent. Using the inequality (15) we obtain

$$
\tilde{P}(s) \leqslant \sum_{j \geqslant 1}\left(e^{2 s C} \sum_{n \in \mathbb{Z}^{*}} e^{-s d\left(x, h^{k n} x\right)} \sum_{m \in \mathbb{Z}^{*}} e^{-s d\left(x, p^{m} x\right)}\right)^{j} .
$$

Because of our choice of $x$, if $l:=d(x, h x)$ then $d\left(x, h^{N} x\right)=|N| l$ for all $N \in \mathbb{Z}$. Thus,

$$
\sum_{n \in \mathbb{Z} *} e^{-s d\left(x, h^{k n} x\right)} \leqslant 2 \frac{e^{-s l k}}{1-e^{-s l k}}
$$

Let $s_{\epsilon}:=\delta_{\mathcal{P}}+\epsilon>\delta_{\mathcal{P}}$ and denoting $P_{s}=\sum_{m \in \mathbb{Z}} e^{-\left(\delta_{\mathcal{P}}+s\right) d\left(x, p^{m} x\right)}$, then the sum $P_{\epsilon}$ is finite. Assuming $\epsilon$ small, we get a constant $D$ such that

$$
e^{2 s_{\epsilon} C_{2}} 2 \frac{e^{-s_{\epsilon} k l}}{1-e^{-s_{\epsilon} k l}} \sum_{m \in \mathbb{Z}^{*}} e^{-s_{\epsilon} d\left(x, p^{m} x\right)}<D e^{-s_{\epsilon} k l} P_{\epsilon} .
$$

Hence, if $\log \left(D P_{\epsilon}\right) / s_{\epsilon} l<k$, then $D e^{-s_{\epsilon} k l} P_{\epsilon}<1$ and therefore $\delta_{\Gamma_{k}} \leqslant s_{\epsilon}$. Observe that the function $t \mapsto \log \left(D P_{t}\right) / s_{t}$ is continuous, decreasing and unbounded in the interval $(0, \eta)$, for any $0<\eta \leqslant 1$. We can then solve the equation $\log \left(D P_{t}\right) / s_{t} l=$ $k-1$, where $t \in\left(\delta_{p}, \delta_{p}+\eta\right)$ and $k$ is large enough. We call this solution $\epsilon_{k}$. By construction $\delta_{\Gamma_{k}} \leqslant s_{\epsilon_{k}}$. It follows from the definition of $\epsilon_{k}$ that $\lim _{n \rightarrow \infty} P_{\epsilon_{k}}=\infty$. Observe that

$$
\frac{k}{\sum_{\gamma \in \mathcal{P}} e^{-\delta_{\Gamma_{k}} d(x, \gamma x)}} \leqslant \frac{k}{P_{\epsilon_{k}}}=\frac{\log \left(D P_{\epsilon_{k}}\right) /\left(s_{\epsilon_{k}} l\right)+1}{P_{\epsilon_{k}}},
$$

but the RHS goes to 0 as $k \rightarrow \infty$. Since $p$ is of divergence type, it follows from $\left[\mathrm{DOP}\right.$, Theorem A] that $\Gamma_{n}$ is of divergence type.

We proceed to show an explicit family of measures satisfying the property claimed in the second part of Theorem 1.2. We remark that the measures constructed in Theorem 5.1 can not be used at this point, since a compact set in $T^{1} X / \Gamma$ is not necessarily a compact set in the topology of $Y$. Hence, the fact that $\int \tau d \mu_{n} \rightarrow \infty$ does not imply that $\nu_{n} \rightarrow 0$. Despite this difficulty, we can use the geometry to construct the desired family.

Denote by $p$ a parabolic isometry in the generator set $\mathcal{A}$ with maximal critical exponent, that is $\delta_{p, \max }=\delta_{p}$. Take $\Gamma_{n}=<p, h^{n}>$ as in Proposition 5.3. Let $m_{n}^{B M}$ be the Bowen-Margulis measure on $T^{1} X / \Gamma_{n}$. Since an extended Schottky group is a geometrically finite group, the measure $m_{n}^{B M}$ is finite [DOP, Theorem B]. Moreover, it maximises the entropy of the geodesic flow on $T^{1} X / H_{n}$ [OP, Theorem 2]. In other words $h_{m_{n}^{B M}}(g)$ equals $\delta_{\Gamma_{n}}$. Recall that the critical exponent $\delta_{\Gamma_{n}}$ converges to $\delta_{p, \max }$ as $n$ goes to infinity, therefore

$$
h_{m_{n}^{B M}}\left(\left(g_{t}\right)\right) \rightarrow \delta_{p, \max } .
$$

Using the coding property, we know that $T^{1} X / \Gamma_{n}$ (except vectors defining geodesics pointing to the $\Gamma_{n}$-orbit of the fixed points of $h$ and $\left.p\right)$ is identified with $Y_{n}=\left\{(x, t) \in \Sigma_{n} \times \mathbb{R}: 0 \leqslant t \leqslant \tau(x)\right\} / \sim$, where

$$
\Sigma_{n}=\left\{\left(a_{i}^{m_{i}}\right)_{i \in \mathbb{Z}}: a_{i} \in\left\{p, h^{n}\right\}, m_{i} \in \mathbb{Z}\right\},
$$

and the geodesic flow is conjugated to the suspension flow on $\left(Y_{n}, \tau\right)$ (same $\tau$ as before, but for this coding). It is convenient to think $\left(\Sigma_{n}, \sigma\right)$ as a sub-shift of $(\Sigma, \sigma)$. 
Since the Bowen-Margulis measure $m_{n}^{B M}$ is ergodic and has positive entropy it need to be supported in $Y_{n}$ under the corresponding identification, i.e. in the space of geodesics modeled by the suspension flow. In particular we can consider $m_{n}^{B M}$ as supported in some invariant subset of $Y$. Let us call $\nu_{n}^{B M}$ the image measure of $m_{n}^{B M}$ induced by the inclusion $Y_{n} \hookrightarrow Y$ and normalized so that $\nu_{n}^{B M}$ is a probability measure. Observe that (16) implies that

$$
\lim _{n \rightarrow \infty} h_{\nu_{n}}(g)=\delta_{p, \max }
$$

We just need to prove that $\nu_{n}^{B M} \rightarrow 0$ to end the proof of Theorem 1.2. This sequence actually dissipates through the cusp associated to the parabolic element $p$. Recall that $\xi_{p}$ denotes the fixed point of $p$ at infinity. Define $N_{\xi_{p}}(s):=\{x \in X$ : $\left.B_{\xi_{p}}(o, x)>s\right\}$, where $o \in X$ is a reference point. Since $\Gamma$ is geometrically finite, for $s$ large enough $N_{\xi_{p}}(s) /<p>$ embeds isometrically into $T^{1} X / \Gamma$, i.e. it is a standard model for the cusp at $\xi_{p}$. By definition, the group $<p>$ acts co-compactly on $\Lambda_{\Gamma} \backslash\left\{\xi_{p}\right\}$. In other words, if we consider a fundamental domain for the action of $\mathcal{P}$ on $\Lambda_{\Gamma} \backslash\left\{\xi_{p}\right\}$, say $D$, then $\Lambda_{\Gamma} \cap D$ is relatively compact in $D$. Clearly the other fundamental domains are given by $\gamma D$ where $\gamma \in \mathcal{P}$.

In $[D O P]$ it is proven that for any geometrically finite group $\Gamma$ the BowenMargulis measure in the cusp $C$ satisfies a bound of the type

$$
\frac{1}{A_{\Gamma, C}} \sum_{p \in \mathcal{P}} d(x, p x) e^{-\delta_{\Gamma} d(x, p x)} \leqslant m_{B M}^{\Gamma}\left(T^{1} C\right) \leqslant A_{\Gamma, C} \sum_{p \in \mathcal{P}} d(x, p x) e^{-\delta_{\Gamma} d(x, p x)} .
$$

Here the point $x$ is chosen inside $C$ and the constant $A_{\Gamma, C}$ basically depend on the size of $C$ and the minimal distance between $\Lambda_{\Gamma} \bigcap D$ and $\partial D$.

Define $\mathcal{Q}_{i}=N_{\xi_{p}}\left(s_{i}\right) /<p>$, where the sequence $\left\{s_{i}\right\}_{i \geqslant 1}$ is increasing with $\lim _{i} s_{i}=\infty$. We assume $Q_{1}$ provides a standard cusp neighborhood. Denote by $p_{n}$ the projection

$$
p_{n}: T^{1} X / \Gamma_{n} \rightarrow T^{1} X / \Gamma
$$

induced by the inclusion at the level of groups. By definition

$$
\nu_{n}^{B M}=\frac{1}{m_{n}^{B M}\left(T^{1} X / \Gamma_{n}\right)}\left(p_{n}\right)_{*} m_{n}^{B M} .
$$

We will prove that $\lim _{n \rightarrow \infty} \nu_{n}^{B M}\left(T^{1}\left((X / \Gamma) \backslash \mathcal{Q}_{i}\right)\right)=0$ for any $i$. For this it is enough to prove the limit

$$
\lim _{n \rightarrow \infty} \frac{m_{n}^{B M}\left(p_{n}^{-1} T^{1}\left((X / \Gamma) \backslash \mathcal{Q}_{i}\right)\right)}{m_{n}^{B M}\left(p_{n}^{-1} T^{1} \mathcal{Q}_{i}\right)}=0 .
$$

Observe that, if $\pi_{n}: X / \Gamma_{n} \rightarrow X / \Gamma$ is the natural projection, then the sets $\pi_{n}^{-1} \mathcal{Q}_{i}$ are represented by the same one in the universal covering. We denote $S_{i}$ this cusp neighborhood.

Lemma 5.4. The measure $\left.m_{n}^{B M}\left(p_{n}^{-1} T^{1}\left((X / \Gamma) \backslash \mathcal{Q}_{i}\right)\right)\right)$ growth at most linearly in $k_{n}$, that is for certain positive constant $C_{i}$, we have

$$
m_{n}^{B M}\left(p_{n}^{-1} T^{1}\left((X / \Gamma) \backslash \mathcal{Q}_{i}\right)\right) \leqslant C_{i} n .
$$

Proof. Let $D_{0}$ (resp. $D_{n}$ ) be the fundamental domain of $\Gamma$ (resp. $\Gamma_{n}$ ) containing $o \in X$. By definition of fundamental domain, there exists a set $T_{n} \subset \Gamma$ such that

(1) for any $\gamma_{1}, \gamma_{2} \in T_{n}$ and $\gamma_{1} \neq \gamma_{2}$, we have $\gamma_{1} \operatorname{int}\left(D_{0}\right) \cap \gamma_{2} \operatorname{int}\left(D_{0}\right)=\varnothing$, and

(2) $\bigcup_{\gamma \in T_{n}} \gamma D_{0}=D_{n}$. 
Denote by $K_{i}$ the compact $K_{i}=(X / \Gamma) \backslash \mathcal{Q}_{i}$ and let $\widetilde{K}_{i}$ the lift of $K_{i}$ into $X$ intersecting $D_{0}$. By definition, any lift of $K_{i}$ into $X$ intersecting $D_{n}$ is a translation of $\widetilde{K}_{i}$ by an element in $T_{n}$. Since $m_{n}^{B M}$ is supported in $\Lambda_{\Gamma_{n}}$, the Bowen-Margulis measure $\tilde{m}_{n}^{B M}$ on $X$ satisfies

$$
m_{n}^{B M}\left(p_{n}^{-1} T^{1}\left(K_{i}\right)\right) \leqslant \sum_{\substack{\gamma \in T_{n} \\ \gamma \widetilde{K}_{i} \cap C\left(\Gamma_{n}\right) \neq \varnothing}} \tilde{m}_{n}^{B M}\left(T^{1}\left(\gamma \widetilde{K}_{i}\right)\right),
$$

where $C\left(\Gamma_{n}\right)$ is the convex hull of $L\left(\Gamma_{n}\right) \times L\left(\Gamma_{n}\right)$ in $X \cup \partial X$. By construction and convexity of the domains $C_{\gamma}$, there exists a positive integer $N \geqslant 1$ such that

$$
\#\left\{\gamma \in T_{n}: \gamma \widetilde{K}_{i} \cap C\left(\Gamma_{n}\right) \neq \varnothing\right\} \leqslant N n .
$$

In particular, we have

$$
m_{n}^{B M}\left(p_{n}^{-1} T^{1}\left(K_{i}\right)\right) \leqslant N n \tilde{m}_{n}^{B M}\left(T^{1}\left(\widetilde{K}_{i}\right)\right) .
$$

But again, by estimates given in [DOP], the measure $\tilde{m}_{n}^{B M}\left(T^{1}\left(\tilde{K}_{i}\right)\right)$ satisfies

$$
m_{n}^{B M}\left(T^{1}\left(\widetilde{K}_{i}\right)\right) \leqslant L_{i},
$$

where $L_{i}$ is a constant depending on the diameter of $\widetilde{K}_{i}$. By setting $C_{i}=N L_{i}$, the conclusion follows.

Using the comments just below equation (17) we know that the constants $A_{H_{n}, \mathcal{Q}_{i}}$ can be all considered equal to $A_{H_{1}, \mathcal{Q}_{i}}$. We have then

$$
m_{n}^{B M}\left(T^{1} S_{i}\right) \asymp_{A_{H_{1}, \mathcal{Q}_{i}}} \sum_{p \in \mathcal{P}} d(x, p x) e^{-\delta_{\Gamma_{n}} d(x, p x)} .
$$

Hence, from (18) and (19), we get

$$
\begin{aligned}
\frac{m_{n}^{B M}\left(p_{n}^{-1} T^{1}\left(X \backslash \mathcal{Q}_{i}\right)\right)}{m_{n}^{B M}\left(p_{n}^{-1} T^{1} \mathcal{Q}_{i}\right)} & \leqslant \frac{A_{H_{1}, \mathcal{Q}_{i}} C_{i} n}{\sum_{p \in \mathcal{P}} d(x, p x) e^{-\delta_{\Gamma_{n}}} d(x, p x)} \\
& \leqslant \frac{C_{i}^{\prime} n}{\sum_{p \in \mathcal{P}} e^{-\delta_{\Gamma_{n}} d(x, p x)}} .
\end{aligned}
$$

Finally, property (3) in Proposition 5.3 implies that the last term above converges to 0 . Therefore

$$
\lim _{n \rightarrow \infty} \frac{m_{n}^{B M}\left(p_{n}^{-1} T^{1}\left(X \backslash \mathcal{Q}_{i}\right)\right)}{m_{n}^{B M}\left(p_{n}^{-1} T^{1} \mathcal{Q}_{i}\right)}=0,
$$

which concludes the proof of Theorem 1.2.

Corollary 5.5. Let $X$ and $\Gamma$ as in Theorem 1.2. Then, the entropy at infinity of the geodesic flow satisfies

$$
h_{\infty}\left(T^{1} X / \Gamma,\left(g_{t}\right)\right)=\delta_{p, \max } .
$$




\section{ThermodynAmic Formalism}

In this section we always consider $X$ a Hadamard manifold with pinched negative sectional curvature and $\Gamma$ an extended Schottky group of isometries of $X$ with property $(\star)$. We also assume that the derivatives of the sectional curvature are uniformly bounded. Our goal is to obtain several results on thermodynamic formalism for the geodesic flow over $X / \Gamma$. Some of these results were already obtained, without symbolic methods, by Coudène (see [Cou]) and Paulin, Pollicott and Schapira (see [PPS]). However, the strength of our symbolic approach will be clear in the study of regularity properties of the pressure (sub-section 6.2).

Here we keep the notation of sub-section 4.2. Thus, the geodesic flow $\left(g_{t}\right)$ in the set $\Omega_{0}$ can be coded by a suspension semi-flow $(Y, \Phi)$ with base $(\Sigma, \sigma)$ and roof function $\tau: \Sigma \rightarrow \mathbb{R}$.

6.1. Equilibrium measures. We will consider the following class of potentials.

Definition 6.1. A continuous function $f: T^{1} X / \Gamma \rightarrow \mathbb{R}$ belongs to the class of regular functions, that we denote by $\mathcal{R}$, if the symbolic representation $\Delta_{f}: \Sigma \rightarrow \mathbb{R}$ of $\left.f\right|_{\Omega_{0}}$ has summable variations.

We begin studying thermodynamic formalism for the geodesic flow restricted to the set $\Omega_{0}$. The following results can be deduced from the general theory of suspension flows over countable Markov shifts and from the symbolic model for the geodesic flow. With a slight abuse of notation, using the indentification explicited before, we still denote by $f: Y \rightarrow \mathbb{R}$ the given map $f: \Omega_{0} \rightarrow \mathbb{R}$.

Definition 6.2. Let $f \in \mathcal{R}$, then the pressure of $f$ with respect to the geodesic flow $g:=\left(g_{t}\right)$ restricted to the set $\Omega_{0}$ is defined by

$$
P_{\Omega_{0}}(f):=\lim _{t \rightarrow \infty} \frac{1}{t} \log \left(\sum_{\varphi_{s}(x, 0)=(x, 0), 0<s \leqslant t} \exp \left(\int_{0}^{s} f\left(\varphi_{k}(x, 0)\right) \mathrm{d} k\right) \chi_{C_{i_{0}}}(x)\right) .
$$

This pressure satisfies the following properties:

Proposition 6.3 (Variational Principle). Let $f \in \mathcal{R}$, then

$$
P_{\Omega_{0}}(f)=\sup \left\{h_{\nu}(g)+\int_{\Omega_{0}} f \mathrm{~d} \nu: \nu \in \mathcal{M}_{\Omega_{0}} \text { and }-\int_{\Omega_{0}} f \mathrm{~d} \nu<\infty\right\},
$$

where $\mathcal{M}_{\Omega_{0}}$ denotes the set of $\left(g_{t}\right)$-invariant probability measures supported in $\Omega_{0}$.

Proposition 6.4. Let $f \in \mathcal{R}$, then

$$
P_{\Omega_{0}}(f)=\sup \left\{P_{g \mid K}(f): K \in \mathcal{K}_{\Omega_{0}}(g)\right\},
$$

where $\mathcal{K}_{\Omega_{0}}(g)$ denotes the space of compact $g$-invariant sets in $\Omega_{0}$.

Remark 6.5 (Convexity). It is well known that for any $K \in \mathcal{K}_{\Omega_{0}}(g)$ the pressure function $P_{g \mid K}(\cdot)$ is convex. Since the supremum of convex functions is a convex function, it readily follows that $P_{\Omega_{0}}(\cdot)$ is convex.

Proposition 6.6. Let $f \in \mathcal{R}$. Then there is an equilibrium measure $\nu_{f} \in \mathcal{M}_{\Omega_{0}}$, that is,

$$
P_{\Omega_{0}}(f)=h_{\nu_{f}}(g)+\int_{\Omega_{0}} f \mathrm{~d} \nu_{f}
$$


for $f$ if and only if we have that $P_{\sigma}\left(\Delta_{f}-P_{\Phi}(f) \tau\right)=0$ and there exists an equilibrium measure $\mu_{f} \in \mathcal{M}_{\sigma}$ for $\Delta_{f}-P_{\Phi}(f) \tau$ such that $\int \tau d \mu_{f}<\infty$. Moreover, if such an equilibrium measure exists then it is unique.

In order to extend these results to the geodesic flow in $T^{1} X / \Gamma$ we use the second conclusion of Proposition 4.10.

Definition 6.7. Let $f \in \mathcal{R}$, then the pressure of $f$ with respect to the geodesic flow $g:=\left(g_{t}\right)$ in $T^{1} X / \Gamma$ is defined by

$$
P_{g}(f):=\max \left\{P_{\Omega_{0}}(f), \int f \mathrm{~d} \nu^{h_{1}}, \ldots, \int f \mathrm{~d} \nu^{h_{N_{1}}}\right\} .
$$

Proposition 6.8 (Variational Principle). Let $f \in \mathcal{R}$, then

$$
P_{g}(f)=\sup \left\{h_{\nu}(g)+\int f \mathrm{~d} \nu: \nu \in \mathcal{M}_{g} \text { and }-\int f \mathrm{~d} \nu<\infty\right\},
$$

Proposition 6.9. Let $f \in \mathcal{R}$, then

$$
P_{g}(f)=\sup \left\{P_{g \mid K}(f): K \in \mathcal{K}(g)\right\},
$$

where $\mathcal{K}(g)$ denotes the space of compact $g$-invariant sets.

Proposition 6.10. Let $f \in \mathcal{R}$ be such that $\sup f<P_{g}(f)$ then there is an equilibrium measure $\nu_{f} \in \mathcal{M}_{g}$, for $f$ if and only if we have that $P_{\Omega_{0}}\left(\Delta_{f}-P_{\Phi}(f) \tau\right)=0$ and there exists an equilibrium measure $\mu_{f} \in \mathcal{M}_{\sigma}$ for $\Delta_{f}-P_{\Phi}(f) \tau$ such that $\int \tau d \mu_{f}<\infty$. Moreover, if such an equilibrium measure exists then it is unique.

Proof. Note that if $\sup f<P_{g}(f)$ then an equilibrium measure for $f$, if it exists, must have positive entropy. Since the measures $\nu^{h_{i}}$, with $i \in\left\{1, \ldots, N_{1}\right\}$ have zero entropy (see Proposition 4.10). The result follows from Proposition 6.6.

The next result shows that potentials with small oscillation do have equilibrium measures, this result can also be deduced from [Cou, PPS]. Our proof is short and uses the symbolic structure.

Theorem 6.11. Let $f \in \mathcal{R}$. If

$$
\sup f-\inf f<h_{\text {top }}(g)-\delta_{p, \max }
$$

then $f$ has an equilibrium measure.

Proof. Assume that the measures $\nu^{h_{i}}$ are not equilibrium measures for $f$, otherwise the theorem is proved. Therefore, we have that $P_{g}(f)=P_{\Omega_{0}}(f)$. We first show that $P_{\sigma}\left(\Delta_{f}-P_{g}(f) \tau\right)=0$. Note that for every $x \in \Sigma$,

$$
\tau(x) \inf f \leqslant \Delta_{f}(x) \leqslant \tau(x) \sup f .
$$

By monotonicity of the pressure we obtain

$$
P_{\sigma}((\inf f-t) \tau) \leqslant P_{\sigma}\left(\Delta_{f}-t \tau\right) \leqslant P_{\sigma}((\sup f-t) \tau) .
$$

Let $t \in\left(s_{\infty}+\sup f, h_{t o p}(g)+\inf f\right)$ and recall that $s_{\infty}=\delta_{p, \max }$. Then

$$
0<P_{\sigma}((\inf f-t) \tau) \leqslant P_{\sigma}\left(\Delta_{f}-t \tau\right) \leqslant P_{\sigma}((\sup f-t) \tau)<\infty .
$$

Since $P_{g}(f)<\infty$ and the function $t \rightarrow P_{\sigma}\left(\Delta_{f}-t \tau\right)$ is continuous with $\lim _{t \rightarrow \infty} P_{\sigma}\left(\Delta_{f}-\right.$ $t \tau)=-\infty$, we obtain that $P_{\sigma}\left(\Delta_{f}-P_{g}(f) \tau\right)=0$. Since the system $\Sigma$ has the BIP 
condition and the potential $\Delta_{f}-P_{g}(f) \tau$ is of summable variations, it has an equilibrium measure $\mu$. It remains to prove the integrability condition. Recall that

$$
\left.\frac{\partial}{\partial t} P_{\sigma}\left(\Delta_{f}-t \tau\right)\right|_{t=P_{g}(f)}=-\int \tau d \mu \text {. }
$$

But we have proved that the function $t \rightarrow P_{\sigma}\left(\Delta_{f}-t \tau\right)$ is finite (at least) in an interval of the form $\left[P_{g}(f)-\epsilon, P_{g}(f)+\epsilon\right]$. The result now follows, because when finite the function is real analytic.

6.2. Phase transitions. This sub-section is devoted to study the regularity properties of pressure functions $t \mapsto P_{g}(t f)$ for a certain class of functions $f$. We say that the pressure function $t \mapsto P_{g}(t f)$ has a phase transition at $t=t_{0}$ if the pressure function is not real analytic at $t=t_{0}$. The set of points at which the pressure function exhibits phase transitions might be a very large set. However, since the pressure is a convex function it can only have a countable set of points where it is not differentiable. Regularity properties of the pressure are related to important dynamical properties, for example exponential decay of correlations of equilibrium measures. In the Axiom A case the pressure is real analytic. Indeed, this can be proved noting that, in that setting, the function $t \mapsto P_{\sigma}\left(\Delta_{f}-t \tau\right)$ is real analytic and that $P_{\sigma}\left(\Delta_{f}-P_{\Phi} \tau\right)=0$. The result then follows from the implicit function theorem noticing that the non-degeneracy condition is fulfilled:

$$
\frac{\partial}{\partial t} P_{\sigma}\left(\Delta_{f}-t \tau\right)=-\int \tau d \mu<0
$$

where $\mu$ is the equilibrium measure corresponding to $\Delta_{f}-t \tau$. The inequality above, together with the coding properties established in [Bo1, BR, Ra], allow us to establish that the pressure is real analytic for regular potentials in the Axiom A setting. In the non-compact case the situation can be different. However, the only results involving the regularity properties of the pressure function for geodesic flows defined on non-compact manifolds, that we are aware of, are those concerning the modular surface (see [IJ, Section 6]). In this section we establish regularity results for pressure functions of geodesic flows defined on extended Schottky groups. We begin by defining conditions (F1) and (F2) on the potentials.

Definition 6.12. Consider a non-negative continuous function $f: T^{1} X / \Gamma \rightarrow \mathbb{R}$. We will say $f$ satisfies Condition (F1) or (F2) if the corresponding property below holds.

(F1) The symbolic representation $\Delta_{f}: \Sigma^{+} \rightarrow \mathbb{R}$ is locally Hölder and bounded away from zero in every cylinder $C_{a^{m}} \subset \Sigma^{+}$, where $a \in \mathcal{A}, m \in \mathbb{Z}$.

(F2) Consider any indexation $\left(C_{n}\right)_{n \in \mathbb{N}}$ of the cylinders of the form $C_{a^{m}}$. Then,

$$
\lim _{n \rightarrow \infty} \frac{\sup \left\{\Delta_{f}(x): x \in C_{n}\right\}}{\inf \left\{\tau(x): x \in C_{n}\right\}}=0 .
$$

We say $f$ belong to the class $\mathcal{F}$ if it satisfies (F1) and (F2).

In the following Lemma we establish two properties of potentials in $\mathcal{F}$ that will be used in the sequel.

Lemma 6.13. Let $f$ be a potential satisfying $(F 1)$ and $\left(\nu_{n}\right)$ a sequence of measures in $\mathcal{M}_{\Omega_{0}}$ such that $\nu_{n}=\frac{\mu_{n} \times\left. m\right|_{Y}}{\left(\mu_{n} \times m\right)(Y)}$. Then,

(1) if $\lim _{n \rightarrow \infty} \int_{\Omega_{0}} f d \nu_{n}=0$, then $\lim _{n \rightarrow \infty} \int \tau d \mu_{n}=\infty$. 
(2) if $f$ satisfies Condition (F2) and $\lim _{n \rightarrow \infty} \int \tau d \mu_{n}=\infty$, then $\lim _{n \rightarrow \infty} \int_{\Omega_{0}} f d \nu_{n}=$ 0 .

Proof. To prove (1) we will argue by contradiction. Assume, passing to a subsequence if necessary, that

$$
\lim _{n \rightarrow \infty} \int \tau d \mu_{n}=C .
$$

Let $\epsilon>0$, there exists $N \in \mathbb{N}$ such that for every $n>N$ we have that

$$
\left|\int \tau d \mu_{n}-C\right|<\epsilon
$$

Lemma 6.14. Let $r>1$ then for every $n>N$ we have that

$$
\mu_{n}(\{x: \tau(x) \leqslant r\})>1-\frac{C+\epsilon}{r} .
$$

Proof of Lemma 6.14. Since the function $\tau$ is positive we have

$$
\int \tau d \mu_{n} \geqslant r \mu_{n}(\{x: \tau(x) \geqslant r\})+\int_{\{x: \tau(x) \leqslant r\}} \tau d \mu_{n} .
$$

Thus,

$$
\begin{aligned}
& C+\epsilon \geqslant r \mu_{n}(\{x: \tau(x) \geqslant r\}) . \\
& \frac{C+\epsilon}{r} \geqslant \mu_{n}(\{x: \tau(x) \geqslant r\}) .
\end{aligned}
$$

Finally

$$
\mu_{n}(\{x: \tau(x) \leqslant r\})>1-\frac{C+\epsilon}{r} .
$$

Note that the set $\{x: \tau(x) \leqslant r\}$ is contained in a finite union of cylinders on $\Sigma$. This follows from the inequality $d\left(o, a^{m} o\right)-C \leqslant \tau(x)$, which is a consequence of Lemma 4.4, and the fact that $\mathcal{A}$ is finite. Since $\Delta_{f}$ is bounded away from zero in every one of them, there exist a constant $G(r)>0$ such that

$$
\Delta_{f}(x)>G(r),
$$

on $\{x: \tau(x) \leqslant r\}$. Thus

$$
\int_{\Omega_{0}} f d \nu_{n}=\frac{\int_{\Sigma} \Delta_{f}(x) d \mu_{n}}{\int_{\Sigma} \tau d \mu_{n}} \geqslant \frac{\int_{\{x: \tau(x) \leqslant r\}} \Delta_{f}(x) d \mu_{n}}{\int_{\Sigma} \tau d \mu_{n}} \geqslant \frac{G(r)\left(1-\frac{C+\epsilon}{r}\right)}{C-\epsilon} .
$$

If we choose $r$ large enough so that $1-\frac{C+\epsilon}{r}>0$ we obtain the desired contradiction.

To prove (2) observe that for every $\varepsilon>0$ there exists $N \geqslant 1$ such that for every $k \geqslant N$, we have

$$
\frac{\sup \left\{\Delta_{f}(x): x \in C_{k}\right\}}{\inf \left\{\tau(x): x \in C_{k}\right\}}<\varepsilon
$$


Hence,

$$
\begin{aligned}
\lim _{n \rightarrow \infty} \int f d \nu_{n} & =\lim _{n \rightarrow \infty} \frac{1}{\int \tau d \mu_{n}} \sum_{k \geqslant 1} \int_{C_{k}} \Delta_{f} d \mu_{n} \\
& =\lim _{n \rightarrow \infty} \frac{1}{\int \tau d \mu_{n}} \sum_{k \geqslant N} \int_{C_{k}} \Delta_{f} d \mu_{n} \\
& \leqslant \lim _{n \rightarrow \infty} \frac{1}{\int \tau d \mu_{n}} \sum_{k \geqslant N} \int_{C_{k}} \frac{\sup \left\{\Delta_{f}(x): x \in C_{k}\right\}}{\inf \left\{\tau(x): x \in C_{k}\right\}} \inf \left\{\tau(x): x \in C_{k}\right\} d \mu_{n} \\
& \leqslant \lim _{n \rightarrow \infty} \frac{1}{\int \tau d \mu_{n}} \sum_{k \geqslant N} \int_{C_{k}} \varepsilon \inf \left\{\tau(x): x \in C_{k}\right\} d \mu_{n} \\
& \leqslant \varepsilon .
\end{aligned}
$$

Since $\varepsilon>0$ is arbitrary, it follows the conclusion of the second claim.

Combining Theorem 3.7 and Lemma 6.13, we obtain the following

Lemma 6.15. Let $\Gamma$ be an extended Schottky group with property $(\star)$ and let $f$ a function satisfying property (F1). If $\left(\nu_{n}\right)_{n} \subset \mathcal{M}_{g}$ is a sequence of invariant probability measure for the geodesic flow such that

$$
\lim _{n \rightarrow \infty} \int_{T^{1} X / \Gamma} f d \nu_{n}=0
$$

Then

$$
\limsup _{n \rightarrow \infty} h_{\nu_{n}}(g) \leqslant \delta_{p, \max } .
$$

Proof. If $\nu_{n} \in \mathcal{M}_{\Omega_{0}}$ the Lemma follows directly from (1) in Lemma 6.13 and Theorem 3.7. If $\nu_{n} \in \mathcal{M}_{g} \backslash \mathcal{M}_{\Omega_{0}}$ then we can consider the measure $\tilde{\nu}_{n}:=\nu_{n}-\sum_{i=1}^{N_{1}} c_{i}^{n} \nu^{h_{i}}$ where the constants $c_{i}^{n} \geqslant 0$ are chosen so that $\tilde{\nu}_{n}$ (periodic orbit associated to hyperbolic generator $\left.h_{i}\right)=0$. Let $C_{n}=\tilde{\nu}_{n}(X / \Gamma)$. If $C_{n}=0$ then $h\left(\nu_{n}\right)=0$ and there is not contribution to the desired $\lim \sup h\left(\nu_{n}\right)$. Otherwise define $u_{n}=C_{n}^{-1} \tilde{\nu}_{n}$. By definition $u_{n}$ is a probability measure in $\mathcal{M}_{\Omega_{0}}$, we claim $\lim _{n \rightarrow \infty} \int f d u_{n}=0$. Observe $\int f d \nu_{n}=\int f d \tilde{\nu}_{n}+\sum_{i=1}^{N_{1}} c_{i}^{n} \nu^{h_{i}}(f)$ has non-negative summands and it is converging to zero, it follows that $\int f d \tilde{\nu_{n}} \rightarrow 0, c_{i}^{n} \rightarrow 0$ as $n \rightarrow \infty$. By definition $C_{n}=1-\sum_{i=1}^{N_{1}} c_{i}^{n}$, therefore $C_{n} \rightarrow 1$. Recalling $u_{n}=C_{n}^{-1} \tilde{\nu}_{n}$ we get $\lim \int f d u_{n}=0$. Because $\nu_{n}=C_{n} u_{n}+\left(1-C_{n}\right)\left(1-C_{n}\right)^{-1} \sum_{i=1}^{N_{1}} c_{i}^{n} \nu^{h_{i}}$, we have $h_{\nu_{n}}(g)=C_{n} h_{u_{n}}(g)$. Finally since $C_{n} \rightarrow 1$ and $\limsup _{n \rightarrow \infty} h_{u_{n}}(g) \leqslant \delta_{p, \max }$ (because $u_{n} \in \mathcal{M}_{\Omega_{0}}$ ), we get $\lim \sup _{n \rightarrow \infty} h_{\nu_{n}}(g) \leqslant \delta_{p, \max }$.

The next Theorem is the main result of this sub-section and it is an adaptation of results obtained at a symbolic level in [IJ]. It is possible to translate those symbolic results into this geometric setting thanks to Theorem 4.12.

Theorem (1.3). Let $X$ be a Hadamard manifold with pinched negative sectional curvature and let $\Gamma$ be an extended Schottky group of isometries of $X$ with property $(\star)$. Assume that the derivatives of the sectional curvature are uniformly bounded. If $f \in \mathcal{F}$, then

(1) For every $t \in \mathbb{R}$ we have that $P_{g}(t f) \geqslant \delta_{p, \max }$.

(2) We have that $\lim _{t \rightarrow-\infty} P_{g}(t f)=\delta_{p, \max }$. 
(3) Let $t^{\prime}:=\sup \left\{t \in \mathbb{R}: P_{g}(t f)=\delta_{p, \max }\right\}$, then

$$
P_{g}(t f)= \begin{cases}\delta_{p, \max } & \text { if } t<t^{\prime} ; \\ \text { real analytic, strictly convex, strictly increasing } & \text { if } t>t^{\prime} .\end{cases}
$$

(4) If $t>t^{\prime}$, the potential tf has a unique equilibrium measure. If $t<t^{\prime}$ it has no equilibrium measure.

Note that Theorem 1.3 shows that when $t^{\prime}$ is finite then the pressure function exhibits a phase transition at $t=t^{\prime}$ whereas when $t^{\prime}=-\infty$ the pressure function is real analytic where defined (see Figure below). Recall that $\delta_{p, \max }=s_{\infty}$.

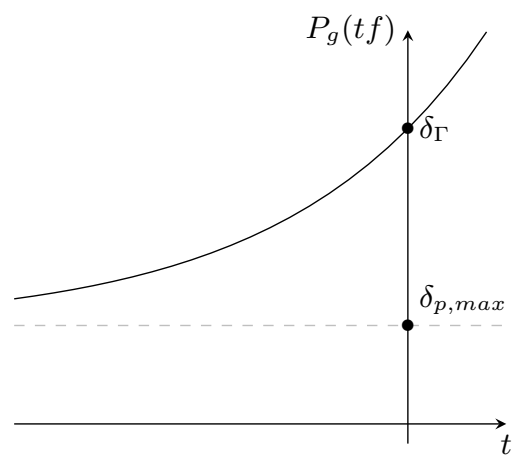

No phase transitions

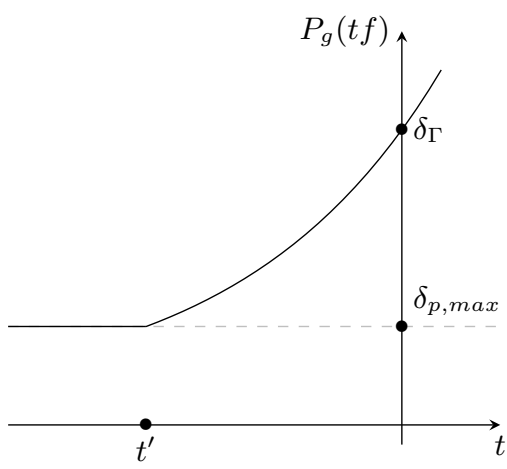

Phase transition at $t=t^{\prime}$

Proof of (1). The first claim follows from the variational principle. By Theorem 5.1 there exists a sequence $\left(\nu_{n}\right) \subset \mathcal{M}_{g}$ such that $\lim _{n \rightarrow \infty} h_{\nu_{n}}(g)=s_{\infty}$ and their corresponding probability $\sigma$-invariant measures $\left(\mu_{n}\right)$ in $\Sigma$ satisfy $\lim _{n \rightarrow \infty} \int \tau d \mu_{n}=$ $\infty$. Therefore, by (2) in Lemma 6.13, we also have that $\lim _{n \rightarrow \infty} \int f d \nu_{n}=0$. Hence, for every $t \in \mathbb{R}$, we have

$$
\begin{aligned}
s_{\infty}=\delta_{p, \max } & =\lim _{n \rightarrow \infty}\left(h_{\nu_{n}}(g)+t \int_{\Omega_{0}} f d \nu_{n}\right) \\
& \leqslant \sup \left\{h_{\nu}(g)+t \int f d \nu: \nu \in \mathcal{M}_{g}\right\}=P_{g}(t f) .
\end{aligned}
$$

Proof of (2). Since $t \mapsto P_{g}(t f)$ is non-decreasing and bounded below, the following

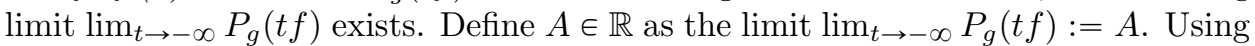
the Variational Principle, we can choose a sequence of measures $\left(\nu_{n}\right)_{n}$ in $\mathcal{M}_{g}$ for which

$$
\lim _{n \rightarrow \infty} h_{\nu_{n}}(g)-n \int f d \nu_{n}=A .
$$

Since $A$ is finite it follows that $\lim _{n \rightarrow \infty} \int f d \nu_{n}=0$. Hence, from Lemma 6.15, we obtain $\lim \sup _{n \rightarrow \infty} h_{\nu_{n}}(g) \leqslant s_{\infty}$. In particular,

$$
\begin{aligned}
s_{\infty} & \leqslant \lim _{t \rightarrow-\infty} P_{g}(t f) \\
& =\lim _{n \rightarrow \infty} h_{\nu_{n}}(g)-n \int f d \nu_{n} \\
& \leqslant \lim _{n \rightarrow \infty} h_{\nu_{n}}(g) \leqslant s_{\infty} .
\end{aligned}
$$


Therefore, we have that $A=\delta_{p, \max }$.

Proof of (3): Real analyticity. We first prove $P_{g}(t f)=P_{\Omega_{0}}(t f)$. After this is done we can proceed with standard regularity arguments in the symbolic picture. Observe that for $t<0$ the pressure $P_{\Omega_{0}}(t f)$ is always positive while the contribution of the pressure on $\left(T^{1} X / \Gamma\right) \backslash \Omega_{0}$ is negative, so $P_{g}(t f)=P_{\Omega_{0}}(t f)$ for every $t \leqslant 0$. Consider now $t>0$. Pick $\nu^{h_{i}}$ as in Proposition 4.10 (see also Definition 6.7). Denote by $x_{-}$(resp. $x_{+}$) the repulsor (attractor) of $h_{i}$ and $\gamma_{h_{i}}$ the geodesic defined by those points. Consider $p$ a parabolic element in $\mathcal{A}$ and let $\gamma_{n}$ be the geodesic connecting the points $\xi^{-}$and $\xi^{+}$where $\omega\left(\xi^{-}\right)=\overline{p^{-1} h^{-n}}$ and $\omega\left(\xi^{+}\right)=\overline{h^{n} p}$. Denote $\gamma_{\infty}$ the geodesic connecting $p^{-1} x_{-}$and $x_{+}$. Observe $\gamma_{n}$ descends to a closed geodesic in $T^{1} X / \Gamma$. By comparing $\gamma_{n}$ and $\gamma_{\infty}$ we see that for any $\epsilon>0$, the amount of time $\gamma_{n}$ leaves a $\epsilon$-neighborhood of $\gamma_{h_{i}}$ is uniformly bounded for big enough $n$. Let $\nu_{n}$ be the invariant probability measure defined by the closed geodesic $\gamma_{n}$, then we get the weak convergence $\nu_{n} \rightarrow \nu^{h_{i}}$. Then

$$
t \int f d \nu^{h_{i}}=\lim _{n \rightarrow \infty} t \int f d \nu_{n} \leqslant \lim _{n \rightarrow \infty}\left(h_{\nu_{n}}(g)+t \int f d \nu_{n}\right) \leqslant P_{\Omega_{0}}(t f) .
$$

This give us $P_{g}(t f)=P_{\Omega_{0}}(t f)$.

The pressure function $t \mapsto P_{g}(t f)$ is convex, non-decreasing and bounded from below by $s_{\infty}$. We now prove that for $t>t^{\prime}$ it is real analytic. Note that since $t^{\prime}<t$ we have that

$$
P_{\sigma}\left(t \Delta_{f}-s_{\infty} \tau\right)>0
$$

possibly infinity and that there exists $p>s_{\infty}$ such that $0<P_{\sigma}\left(t \Delta_{f}-p \tau\right)<\infty$ (see [IJ, Lemma 4.2]). Moreover, Condition (F2) implies that $P_{g}(t f)<\infty$ for every $t>t^{\prime}$, hence

$$
P_{\sigma}\left(\Delta_{t f}-P_{g}(t f) \tau\right) \leqslant 0 .
$$

Since $\tau$ is a positive, the function $s \mapsto P_{\sigma}\left(t \Delta_{f}-s \tau\right)$ is decreasing and

$$
\lim _{s \rightarrow+\infty} P_{\sigma}\left(t \Delta_{f}-s \tau\right)=-\infty
$$

Moreover, since the base map of the symbolic model satisfies the BIP condition, the function $(s, t) \mapsto P_{\sigma}\left(t \Delta_{f}-s \tau\right)$ is real analytic in both variables. Hence, there exists a unique real number $s_{f}>s_{\infty}$ such that $P_{\sigma}\left(t \Delta_{f}-s_{f} \tau\right)=0$ and

$$
\left.\frac{\partial}{\partial s} P_{\sigma}\left(t \Delta_{f}-s \tau\right)\right|_{s=s_{f}}<0 .
$$

Therefore, $P_{g}(t f)=s_{f}$ and by Implicit Function Theorem, the function $t \mapsto P_{g}(t f)$ is real analytic in $\left(t^{\prime}, t^{\star}\right)$.

Proof of (4). First note that the previous claims imply that no zero entropy measure can be an equilibrium measure. Moreover, in the proof of (3) we obtained that for $t \in\left(t^{\prime}, \infty\right)$ we have that $P_{\sigma}\left(t \Delta_{f}-P_{g}(t f) \tau\right)=0$. Since the system satisfies the BIP condition there exists an equilibrium measure $\mu_{f} \in \mathcal{M}_{\sigma}$ for $t \Delta_{f}-P_{g}(t f) \tau$ such that $\int \tau d \mu_{f}<\infty$ (see Theorem 2.9). Therefore it follows from Proposition 6.6 that $t f$ has an equilibrium measure.

In order to prove the last claim, assume by contradiction that for some $t_{1}<t^{\prime}$ the potential $t_{1} f$ has an equilibrium measure $\nu_{t_{1}}$. Then $s_{\infty}=P_{g}\left(t_{1} f\right)=h_{\nu_{t_{1}}}(g)+$ $t_{1} \int_{\Omega_{0}} f d \nu_{t_{1}}$. Since $f>0$ on $\Omega_{0}$, we have that $\int_{\Omega_{0}} f d \nu_{t_{1}}:=B>0$. Thus the straight 
line $r \rightarrow h_{\nu_{t_{1}}}(g)+r \int_{\Omega_{0}} f d \nu_{t_{1}}$ is increasing with $r$, therefore for $t \in\left(t_{1}, t^{\prime}\right)$ we have that

$$
h_{\nu_{t_{1}}}(g)+t \int_{\Omega_{0}} f d \nu_{t_{1}}>s_{\infty}=P_{g}(t f)
$$

This contradiction proves the statement.

6.3. Examples. We will use the following criterion, first introduced in [IJ], to construct phase transitions.

Proposition 6.16. Let $f \in \mathcal{F}$. Then

(1) If there exist $t_{0} \in \mathbb{R}$ such that $P_{\sigma}\left(t_{0} \Delta_{f}-s_{\infty} \tau\right)<\infty$, then there exists $t^{\prime}<t_{0}$ such that for every $t<t^{\prime}$ we have $P_{g}(t f)=s_{\infty}$.

(2) Suppose that there exists an interval I such that $P_{\sigma}\left(t \Delta_{f}-s_{\infty} \tau\right)=\infty$ for every $t \in I$. Then $t \mapsto P_{g}(t f)$ is real analytic on $I$. In particular, if for every $t \in \mathbb{R}$ we have $P_{\sigma}\left(t \Delta_{f}-s_{\infty} \tau\right)=\infty$, then $t \mapsto P_{g}(t f)$ is real analytic in $\mathbb{R}$.

The proof of this Lemma follows as in [IJ, Lemma 4.5, Theorem 4,1]. We now present an example of a phase transition (Example 6.18) and another one with pressure real analytic everywhere (Example 6.19). A useful lemma in order to construct an example of a phase transition, is the following

Lemma 6.17. Let $\left(a_{n}\right)_{n}$ be a sequence of positive real numbers such that $\sum_{n=1}^{\infty} a_{n}^{t}$ converges for every $t>t^{*}$ and diverges at $t=t^{*}$. Then there exists a sequence $\left(\varepsilon_{n}\right)_{n}$ of positive numbers such that $\lim _{n \rightarrow \infty} \varepsilon_{n}=0$ and

$$
\sum_{n=1}^{\infty} a_{n}^{t^{*}+\varepsilon_{n}}<\infty .
$$

Proof of Lemma 6.17. Let $\left(\alpha_{m}\right)_{m}$ any sequence of real numbers in $(0,1]$ converging to zero. Note that, for every $m \geqslant 1$ we have

$$
\sum_{n=1}^{\infty} a_{n}^{t^{*}+\alpha_{m}}<\infty .
$$

Then, there exists an integer $N_{m} \geqslant 1$ such that

$$
\sum_{n=N_{m}}^{\infty} a_{n}^{t^{*}+\alpha_{m}}<1 / m^{2} .
$$


We can suppose without loss of generality that $N_{m}<N_{m+1}$. Define $\varepsilon_{n}$ for every $N_{m} \leqslant n<N_{m+1}$ as $\varepsilon_{n}=\alpha_{m}$, and $\varepsilon_{n}=1$ for $1 \leqslant n<N_{1}$. Thus

$$
\begin{aligned}
\sum_{n=1}^{\infty} a_{n}^{t^{*}+\varepsilon_{n}} & =\sum_{n=1}^{N_{1}-1} a_{n}^{t^{*}+\varepsilon_{n}}+\sum_{m=1}^{\infty} \sum_{n=N_{m}}^{N_{m+1}-1} a_{n}^{t^{*}+\varepsilon_{n}} \\
& =\sum_{n=1}^{N_{1}-1} a_{n}^{t^{*}+1}+\sum_{m=1}^{\infty} \sum_{n=N_{m}}^{N_{m+1}-1} a_{n}^{t^{*}+\alpha_{m}} \\
& \leqslant \sum_{n=1}^{N_{1}-1} a_{n}^{t^{*}+1}+\sum_{m=1}^{\infty} \sum_{n=N_{m}}^{\infty} a_{n}^{t^{*}+\alpha_{m}} \\
& \leqslant \sum_{n=1}^{N_{1}-1} a_{n}^{t^{*}+1}+\sum_{m=1}^{\infty} 1 / m^{2} \\
& <\infty .
\end{aligned}
$$

Example 6.18. (Phase transition) Let $\Gamma$ be a Schottky group with property $(\star)$ and assume that there are at least 2 different cusps, i.e. $N_{2} \geqslant 2$. Moreover assume there exists a unique parabolic generator $p$ with $\delta_{p}=\delta_{p, \max }$. Recall that the series $\sum_{m \in \mathbb{Z}} e^{-\delta_{p} d\left(o, p^{m} o\right)}$ diverges since $p$ is a parabolic isometry of divergence type. Take a decreasing sequence of real numbers $\varepsilon_{m}>0$, as in Lemma 6.17, such that $\lim _{m \rightarrow \infty} \varepsilon_{m}=0$ and $\sum_{m \in \mathbb{Z}} e^{-\left(\delta_{p}+\varepsilon_{m}\right) d\left(o, p^{m} o\right)}<\infty$. Define a function $f^{0}: \Sigma \rightarrow \mathbb{R}^{+}$ by

(1) $f^{0}(x)=\varepsilon_{m} \tau(x)$ if the first symbol of $x$ is $p^{m}$ for some $m \in \mathbb{Z}$.

(2) $f^{0}(x)=1$ otherwise.

Observe that since $\tau$ is locally Hölder, the function $f^{0}$ is also locally Hölder. We first see that $f^{0} \in \mathcal{F}$, for this it is enough to check that Condition (F2) holds. There exist constant $C$ independent of $m$ such that $d\left(o, p^{m} o\right)-C \leqslant \tau(x)$ whenever $x \in C_{p^{m}}$. Then, if $x, y \in C_{p^{m}}$ we have $\tau(x) / \tau(y) \leqslant d\left(o, p^{m} o\right) /\left(d\left(o, p^{m} o\right)-C\right)$, i.e. $\sup _{x \in C_{p} m} \tau(x) / \inf _{x \in C_{p} m} \tau(x)$ is uniformly bounded in $m$, this implies Condition (F2). As shown in [BRW, Section 2], we can construct a continuous function $f: Y \rightarrow \mathbb{R}$ with $\Delta_{f}=f^{0}$. We define $t: \Sigma \rightarrow \mathbb{R}$ by $t(x)=\left(s_{\infty}+\varepsilon_{m}\right)$ if the first symbol of $x$ is $p^{m}$, and $s_{\infty}$ otherwise. By simplicity we will denote $s\left(a^{m}\right)=t(x)$ if the first symbol of $x$ is $a^{m}$. Following notations and ideas of the second part of the proof of Theorem 4.12, we obtain, 


$$
\begin{aligned}
P_{\sigma}\left(-\Delta_{f}-s_{\infty} \tau\right) & =\lim _{n \rightarrow \infty} \frac{1}{n} \log \sum_{x: \sigma^{n} x=x} \exp \left(\sum_{i=0}^{n-1}-\left(\Delta_{f}\left(\sigma^{i} x\right)+s_{\infty} \tau\left(\sigma^{i} x\right)\right)\right) \chi_{C_{h_{1}}}(x) \\
& \leqslant \lim _{n \rightarrow \infty} \frac{1}{n} \log \sum_{x: \sigma^{n} x=x} \exp \left(\sum_{i=0}^{n-1}-\tau\left(\sigma^{i} x\right) t\left(\sigma^{i} x\right)\right) \chi_{C_{h_{1}}}(x) \\
& \leqslant \lim _{n \rightarrow \infty} \frac{1}{n} \log \sum_{a_{1}, \ldots, a_{n}} \sum_{m_{1}, \ldots, m_{n}} \prod_{i=1}^{n} C^{s\left(a_{i}^{m_{i}}\right)} e^{-s\left(a_{i}^{m_{i}}\right) d\left(o, a_{i}^{m_{i}} o\right)} \\
& \leqslant \lim _{n \rightarrow \infty} \frac{1}{n} \log C^{n\left(s_{\infty}+1\right)} \sum_{a_{1}, \ldots, a_{n}} \sum_{m_{1}, \ldots, m_{n}} \prod_{i=1}^{n} e^{-s\left(a_{i}^{m_{i}}\right) d\left(o, a_{i}^{m_{i}} o\right)} \\
& =\lim _{n \rightarrow \infty} \frac{1}{n} \log C^{n\left(s_{\infty}+1\right)}\left(\sum_{a \in \mathcal{A}} \sum_{m \in \mathbb{Z}} e^{-s\left(a^{m}\right) d\left(o, a^{m} o\right)}\right)^{n} \\
& =\log C^{s_{\infty}+1}\left(\sum_{a \in \mathcal{A}} \sum_{m \in \mathbb{Z}} e^{-s\left(a^{m}\right) d\left(o, a^{m} o\right)}\right) .
\end{aligned}
$$

Observe that $\sum_{m} e^{-s\left(a^{m}\right) d\left(o, a^{m} o\right)}$ converges for every $s>\delta_{a}$ and every $a \neq p$. On the other hand, the series $\sum_{m \in \mathbb{Z}} e^{-\left(\delta_{p}+\varepsilon_{m}\right) d\left(o, p^{m} o\right)}$ is finite by construction. In particu$\operatorname{lar} P_{\sigma}\left(-\Delta_{f}-s_{\infty} \tau\right)$ is finite. Observe that $f$ is a potential belonging to the family $\mathcal{F}$, then from Proposition 6.16 it follows that $t \mapsto P_{g}(t f)$ exhibits a phase transition.

Example 6.19. (No phase transition) Let $\Gamma$ be a Schottky group with property $(\star)$. Define $f^{0}: \Sigma \rightarrow \mathbb{R}^{+}$to be constant of value 1 and construct a continuous function $f: Y \rightarrow \mathbb{R}$ with $\Delta_{f}=f^{0}$. Observe

$$
P_{\sigma}\left(t-s_{\infty} \tau\right)=t+P_{\sigma}\left(-s_{\infty} \tau\right)=\infty .
$$

Recall that $P_{\sigma}\left(-s_{\infty} \tau\right)=\infty$, because the maximal parabolic generator is of divergence type (see the first part of Theorem 4.12). Since $\tau$ is unbounded and $f^{0}$ is constant, we can apply Proposition 6.16 to show that $t \mapsto P_{g}(t f)$ is real analytic in $\mathbb{R}$. In particular, it never attains the lower bound $s_{\infty}$.

Remark 6.20. In Example 6.18 and Example 6.19, the potential $f$ is defined (a priori) only on the set $\Omega_{0}$. To extend it continuously to the entire manifold $T^{1} X / \Gamma$, it is enough to define it to be equal to 0 on the complement $\left(T^{1} X / \Gamma\right) \backslash \Omega_{0}$.

\section{REFERENCES}

[Ab] L. M. Abramov, On the entropy of a flow. Dokl. Akad. Nauk SSSR 128 (1959) 873-875.

[AK] W. Ambrose and S. Kakutani, Structure and continuity of measurable flows. Duke Math. J. 9 (1942) 25-42.

[BI] L. Barreira and G. Iommi, Suspension flows over countable Markov shifts. Journal of Statistical Physics 124 no.1, 207-230 (2006).

[Bo1] R. Bowen, Symbolic dynamics for hyperbolic flows, Amer. J. Math. 95 (1973), 429-460.

[Bo2] R. Bowen, Equilibrium states and the ergodic theory of Anosov diffeomorphisms. volume 470 of Lecture Notes in Mathematics. Springer-Verlag, Berlin, revised edition, 2008. With a preface by David Ruelle, Edited by Jean-René Chazottes.

[BR] R. Bowen and D. Ruelle, The ergodic theory of Axiom A flows. Invent. Math. 29 (1975), 181-202. 
[BRW] L. Barreira, L. Radu and C. Wolf, Dimension of measures for suspension flows, Dyn. Syst. 19 (2004), 89107.

[BG] A.I. Bufetov and B.M. Gurevich, Existence and uniqueness of the measure of maximal entropy for the Teichmüller flow on the moduli space of Abelian differentials. Sbornik Mathematics 202 (2011), 935-970.

[BS1] L.A. Bunimovich and Y.G. Sinai, Markov partitions for dispersed billiards. Comm. Math. Phys. 78 (1980/81), 247-280.

[BS2] L.A. Bunimovich and Y.G. Sinai, Statistical properties of Lorentz gas with periodic configuration of scatterers. Comm. Math. Phys. 78 (1980/81), 479-497.

[BuS] J. Buzzi and O. Sarig, Uniqueness of equilibrium measures for countable Markov shifts and multidimensional piecewise expanding maps. Ergodic Theory Dynam. Systems 23 (2003), $1383-1400$.

[Cou] Y. Coudène, Gibbs measures on negatively curved manifolds. J. Dynam. Control Syst. 9 (2003), 89-101.

[DP] F. Dal'bo and M. Peigné, Some negatively curved manifolds with cusps, mixing and counting. Journal für die Reine und Angewandte Mathematik 497 (1998), 141-169.

[DOP] F. Dal'bo, J-P. Otal and M. Peigné, Séries de Poincaré des groupes géométriquement finis. Israel Journal of Mathematics 118 (2000), 109-124.

[Da] Y. Daon, Bernoullicity of equilibrium measures on countable Markov shifts. Discrete Contin. Dyn. Syst. 33 (2013), 4003-4015.

[DGR] L. Díaz, K. Gelfert, and M. Rams, Rich phase transitions in step skew products. Nonlinearity 24 (2011), no. 12, 3391-3412.

[DT] N. Dobbs and M. Todd, Free energy jumps up. Preprint available at arXiv:1512.09245.

[EKP] M. Einsiedler, S. Kadyrov, A. D. Pohl, Escape of mass and entropy for diagonal flows in real rank one situations, arXiv:1110.0910 .

[ELMV] M. Einsiedler, E. Linderstrauss, P. Michel, A. Venkatesh, The distribution of closed geodesics on the modular surface, and Duke's theorem, L'Enseignement Mathématique, 58, Issue 3/4 (2012), 249-313.

[FJLR] A. Fan, T. Jordan, L. Liao and M. Rams, Multifractal analysis for expanding interval maps with infinitely many branches. Trans. Amer. Math. Soc. 367 (2015), no. 3, 18471870.

[Gu B.M. Gurevič, Topological entropy for denumerable Markov chains. Dokl. Akad. Nauk SSSR 10 (1969), 911-915.

[Ha] J. Hadamard, Les surfaces á courbures opposé et leurs lignes géodesiques. J.Math.Pures Appl. (5) 4 (1898), 27-73.

[H] U. Hamenstädt, Symbolic dynamics for the Teichmüller flow. Preprint available at arXiv:1112.6107.

[HI] E. Heintze and H. Im Hof, Geometry of horospheres. J. Differential Geom. vol. 12, Number 4 (1977), 481-491.

[IJ] G. Iommi and T. Jordan, Phase transitions for suspension flows. Comm. Math. Phys. 320 (2013), np. 2, 475-498.

[IJT] G. Iommi, T. Jordan and M. Todd, Recurrence and transience for suspension flows. Israel Journal of Mathematics 209 ,(2015) Issue 2, 547-592.

[IT1] G. Iommi and M. Todd, Natural equilibrium states for multimodal maps. Comm. Math. Phys. 300 (2010), no. 1, 65-94.

[IT2] G. Iommi and M. Todd, Transience in dynamical systems. Ergodic Theory Dynam. Systems 33 (2013), no. 5, 1450-1476.

[JKL] J. Jaerisch, M. Kesseböhmer and S. Lamei, Induced topological pressure for countable state Markov shifts. Stoch. Dyn. 14 (2014), no. 2, 1350016, 31 pp.

[KLKM] S. Kadyrov, E. Lindestrauss, D. Y. Kleinbock, and G. A. Margulis. Entropy in the cusp and singular systems of linear forms. Preprint available at arXiv:1407.5310.

[Ke] T. Kempton, Thermodynamic formalism for suspension flows over countable Markov shifts. Nonlinearity 24 (2011), 2763-2775.

[Led] F. Ledrappier, Entropie et principe variationnel pour le flot géodésique en courbure négative pincée. Monogr. Enseign. Math. 43 (2013), 117-144.

[MU] R. Mauldin and M. Urbański, Graph directed Markov systems: geometry and dynamics of limit sets. Cambridge tracts in mathematics 148, Cambridge University Press, Cambridge 2003. 
[OP] J-P. Otal and M. Peigné, Principe variationnel et groupes kleiniens. Duke Math. J. 125 (2004), no. 1, 15-44.

[PP] W. Parry and M. Pollicott, Zeta Functions and the Periodic Orbit Structure of Hyperbolics Dynamics, Astérisque 187-188, 1990.

[Pa] S.J. Patterson, The limit set of a Fuchsian group. Acta Math. 136 (1976), no. 3-4, 241-273.

[PPS] F. Paulin, M. Pollicott and B. Schapira Equilibrium states in negative curvature. to appear in Astérisque.

[PR] F. Przytycki and J. Rivera-Letelier Geometric pressure for multimodal maps of the interval. Preprint available at arXiv: 1405.2443

[Ra] M. Ratner, Markov partitions for Anosov flows on $n$-dimensional manifolds. Israel J. Math. 15 (1973), 92-114.

[Sa1] O. Sarig, Thermodynamic formalism for countable Markov shifts. Ergodic Theory Dynam. Systems 19 (1999), 1565-1593.

[Sa2] O. Sarig, Phase transitions for countable Markov shifts. Comm. Math. Phys. 217 (2001), $555-577$.

[Sa3] O. Sarig, Thermodynamic formalism for countable Markov shifts. Proc. of Symposia in Pure Math. 89 (2015), 81-117.

[Sav] S. Savchenko, Special flows constructed from countable topological Markov chains. Funct. Anal. Appl. 32 (1998), 32-41.

[Si] Y.G. Sinai. Gibbs measures in ergodic theory (English translation). Russian Math. Surveys 27 (1972) 21-64.

[Su] D. Sullivan, Entropy, Hausdorff measures old and new, and limit sets of geometrically finite Kleinian groups. Acta Math. 153 (1984), no. 3-4, 259-277.

[Wa] P. Walters, An Introduction to Ergodic Theory. Graduate Texts in Mathematics 79, Springer, 1981.

Facultad de Matemáticas, Pontificia Universidad Católica de Chile (PUC), Avenida Vicuña Mackenna 4860, Santiago, Chile

E-mail address: giommi@mat.puc.cl

$U R L:$ http://http://www.mat.uc.cl/ giommi/

IRMAR-UMR 6625 CNRS, Université de Rennes 1, Rennes 35042, France

E-mail address: felipe.riquelme@univ-rennes1.fr

Princeton University, Princeton NJ 08544-1000, USA.

E-mail address: avelozo@math.princeton.edu 